\title{
A HIGHER ORDER NUMERICAL METHOD FOR 3-D DOUBLY PERIODIC ELECTROMAGNETIC SCATTERING PROBLEMS*
}

\author{
MICHAEL J. NICHOLAS ${ }^{\dagger}$
}

\begin{abstract}
We develop a method for 3D doubly periodic electromagnetic scattering. We adapt the Müller integral equation formulation of Maxwell's equations to the periodic problem, since it is a Fredholm equation of the second kind. We use Ewald splitting to efficiently calculate the periodic Green's functions. The approach is to regularize the singular Green's functions and to compute integrals with a trapezoidal sum. Through asymptotic analysis near the singular point, we are able to identify the largest part of the smoothing error and to subtract it out. The result is a method that is third order in the grid spacing size. We present results for various scatterers, including a test case for which exact solutions are known. The implemented method does indeed converge with third order accuracy. We present results for which the method successfully resolves Wood's anomaly resonances in transmission.
\end{abstract}

Key words. periodic electromagnetic scattering, periodic Helmholtz Green's function, singular integrals, Ewald summation, photonic crystals, Wood's anomaly

AMS subject classifications. 00A69, 74J20, 32A55

\section{Introduction}

We present an accurate numerical method for scattering of 3D electromagnetic waves by doubly periodic structures. The method is an intuitively simple numerical scheme based on a boundary integral formulation. This scheme involves smoothing the singular Green's functions in the integrands and finding correction terms to the resulting smooth integrals. The analytical method is based on the singular integral methods of J. Thomas Beale $[4,6,5]$, while the scattering problem is motivated by the work of Stephanos Venakides, Mansoor Haider, Stephen Shipman, and Andrew Barnes $[31,17,27,26,28,3]$. Periodic scattering problems are of interest to electrical engineers and physicists who apply them to photonic crystal lattices [11, 24, 14, 18, 29]. The propagation of waves through such lattices is sensitive to the crystals' geometry, their material properties, and on the nature of the incident waves. The recent numerical and experimental discoveries of resonances in transmission have generated interest in periodic electromagnetic scattering problems [12, 34, 15]. Much numerical work investigating these problems and resonances has been done in the $2 \mathrm{D}$ case $[31,17,27,26]$, but less has been done in 3D. The problem was previously solved in 3D by Barnes [3], who used a boundary element method to approximate solutions with $O(h)$ accuracy. Our method is more accurate. A higher order method is desirable in order to investigate resonance peaks in transmission. Boundary integral methods in periodic scattering problems must deal with two numerical issues: (1) The periodic Green's function is an infinite series that converges too slowly to be of practical use, and (2) singularities in the integrals can cause low accuracy in basic numerical schemes. Ewald summation for the Green's functions is commonly used to overcome the first of these obstacles. For us, it also leads to a natural regularization and correction technique, allowing for higher order approximation of the singular integrals. Boundary integral methods in scattering problems are usually rather involved and use

\footnotetext{
*Received: April 1, 2008; accepted (in revised version): July 2, 2008. Communicated by Lenya Ryzhik.

†Mathematics Department, Tulane University, 6823 St. Charles Ave., New Orleans, Louisiana, 70118 (mnichol@tulane.edu).
} 
boundary elements $[3,24,11,33]$. The method developed here is straightforward in implementation. In solving these problems, we have used the Müller integral equation formulation [20], since it is a Fredholm integral equation of the second kind and is well posed. Müller derived his equations for the case of a compact scatterer. We outline the derivation and adapt it to a periodic scatterer in Sec. 4. The Müller formulation involves various singular integrals of surface currents. For each of these, we replace the Green's functions with regularized, but still periodic, counterparts. A smoothing parameter $\delta$ controls the radius of the smoothing, and we set it to be the order of the grid spacing $h$ in size. The smoothing has a local effect like replacing a $1 / r$ singularity with the smooth function erf $(r / \delta) / r$. In Sec. 6 , we perform a local analysis of the singularity in order to identify the largest sources of error introduced by the smoothing. The derivation involves transforming to principal directions using concepts from differential geometry. The correction terms are necessarily invariant under this transformation and depend on geometric properties of the scatterer such as the mean curvature and the differential of the Gauss map. From this analysis, we derive corrections which, when added to the smoothed integrals, reduce the smoothing error to $O\left(\delta^{3}\right)$. Estimates exist that show the discretization error in numerically evaluating these integrals is also small [5]. The scattering problem is exactly solvable for a flat slab scatterer. This provides a test case for our method. Numerical results showing excellent agreement for the test case are given in Sec. 8. Sec. 8 includes results on various curved scatterers as well. In all cases, third order convergence is observed. We also present results in which certain resonances known as Wood's anomalies are resolved very nicely. Wood's anomalies have been observed experimentally [32, 34, 15] and are present at certain frequencies at which singularities in the Green's functions exist. Our Wood's anomaly resonances are unique from previously known anomalies in that they occur near singularities of the interior Green's function instead of the exterior Green's function.

2. Electromagnetic scattering. In a scattering experiment, an object called the scatterer is introduced to a medium in which traveling electromagnetic waves of frequency $\omega$ are present. The scatterer is a domain $\Omega$ in $\mathbb{R}^{3}$ whose electromagnetic properties differ from those of the outside medium. The goal of the problem is to find the resulting electric and magnetic fields ( $\mathbf{E}$ and $\mathbf{H}$, respectively) in all of $\mathbb{R}^{3}$.

The electric permittivity and magnetic permeability of materials are represented respectively as $\epsilon$ and $\mu$ and have different values in $\Omega$ than in its complement $\Omega^{C}$. In $\Omega$ we will call these $\epsilon_{i n t}$ and $\mu_{i n t}$. In $\Omega^{C}$ they will be $\epsilon_{\text {ext }}$ and $\mu_{\text {ext }}$. The ratio $\epsilon_{\text {int }} / \epsilon_{\text {ext }}$ is known as the dielectric contrast of the problem. Materials for which $\epsilon_{i n t}$ is real-valued are known as lossless materials, and materials for which $\epsilon_{i n t}$ has an imaginary part are called lossy. In keeping with current research [31, 17, 27], we will take $\epsilon_{e x t}=\mu_{e x t}=\mu_{i n t}=1$ for the bulk of this paper. The method here presented, however, is valid for general constants.

We assume $t$ dependence of the form $e^{-i \omega t}$ in the fields. Then, Maxwell's equations reduce to the Helmholtz equation:

$$
\Delta \mathbf{E}+k^{2}(\mathbf{x}) \mathbf{E}=0 \quad \Delta \mathbf{H}+k^{2}(\mathbf{x}) \mathbf{H}=0
$$

for

$$
k^{2}(\mathbf{x})=\left\{\begin{array}{l}
k_{i n t}^{2}=\omega^{2} \epsilon_{i n t} \mu_{i n t} \text { for } \mathbf{x} \in \Omega \\
k_{e x t}^{2}=\omega^{2} \epsilon_{e x t} \mu_{e x t} \text { for } \mathbf{x} \notin \Omega
\end{array}\right.
$$


We will distinguish various fields with subscripts. The fields $\mathbf{E}_{i n c}$ and $\mathbf{H}_{i n c}$ are the incident fields that would exist in the absence of the scatterer. Subscripts int and ext refer respectively to the fields in and out of $\Omega$. Fields $\mathbf{E}_{\text {scatt }}$ and $\mathbf{H}_{\text {scatt }}$ are scattered fields and are defined outside of $\Omega$ as $\mathbf{E}_{\text {scatt }}=\mathbf{E}_{\text {ext }}-\mathbf{E}_{\text {inc }}$ and $\mathbf{H}_{\text {scatt }}=\mathbf{H}_{\text {ext }}-\mathbf{H}_{\text {inc }}$.

For our numerical method, the surface of the scatterer $\partial \Omega$ must be in $C^{3}$. The reasons for this will become clear in Sec. 6 .

2.1. 3D doubly periodic scatterers. We will work in $\mathbb{R}^{3}$ and will use scatterers $\Omega$ that are $2 \pi$ periodic in the $x$ and $y$ directions and of finite thickness in the $z$ direction. Generalization to any period is not difficult.

Our method allows us to do all of our calculations on just one period in both $x$ and $y$ of the scatterer. From this point on, we will let $\Omega$ and $\partial \Omega$ refer to just one periodic block of the scatterer.

2.2. The scattering problem. Our problem is to solve (2.1) in the presence of the scatterer. We require that boundary conditions of tangential continuity are satisfied on $\partial \Omega$ :

$$
\mathbf{n} \times \mathbf{E}_{\text {ext }}=\mathbf{n} \times \mathbf{E}_{\text {int }} \quad \mathbf{n} \times \mathbf{H}_{\text {ext }}=\mathbf{n} \times \mathbf{H}_{\text {int }}
$$

where $\mathbf{n}$ is the outward unit normal to $\Omega$. Our fields must also satisfy radiation conditions, which ensure that the fields propagate and decay properly while maintaining periodicity. As $z \rightarrow \pm \infty$, we require the scattered fields to have the form

$$
\begin{aligned}
& \mathbf{E}=\sum_{m, n} \mathbf{E}_{m n}^{ \pm} e^{-\sqrt{-\lambda_{m n}}|z|} e^{i(m+\alpha) x+i(n+\beta) y}, \\
& \mathbf{H}=\sum_{m, n} \mathbf{H}_{m n}^{ \pm} e^{-\sqrt{-\lambda_{m n}}|z|} e^{i(m+\alpha) x+i(n+\beta) y} .
\end{aligned}
$$

Throughout this paper, all sums of this form are from $-\infty$ to $\infty$. Here $\lambda_{m n}=$ $k^{2}-(m+\alpha)^{2}-(n+\beta)^{2}$, and $\mathbf{E}_{m n}^{ \pm}$and $\mathbf{H}_{m n}^{ \pm}$are constant vectors. The constants $\alpha$ and $\beta$ depend on the fields' angles of incidence and are defined in Sec. 2.4. These radiation conditions specify large $z$ behavior of the Fourier modes of the fields. The negative sign in the exponentials ensures that the modes decay whenever $\operatorname{Re}\left(\sqrt{-\lambda_{m n}}\right)$ is nonzero. If, however, $\operatorname{Re}\left(\sqrt{-\lambda_{m n}}\right)=0$, we must choose the negative square root instead of the principal square root so that the waves propagate in the correct direction (from negative to positive $z$ ). That is, we must choose $\operatorname{Im}\left(\sqrt{-\lambda_{m n}}\right)<0$. We adopt the same sign conventions for the modes of the Green's function that will be introduced in Sec. 3.

2.3. The incident wave. We will always take the incident wave to be a plane wave propagating from the negative $z$ to positive $z$ direction. The direction of the incident wave is given by the unit vector $\vec{\gamma}=\left(\gamma_{1}, \gamma_{2}, \gamma_{3}\right)$. With azimuthal angle $\theta$ and polar angle from the $z$-axis $\phi, \vec{\gamma}$ is

$$
\left(\begin{array}{l}
\gamma_{1} \\
\gamma_{2} \\
\gamma_{3}
\end{array}\right)=\left(\begin{array}{c}
\cos \theta \sin \phi \\
\sin \theta \sin \phi \\
\cos \phi
\end{array}\right)
$$

with $0 \leq \phi<\frac{\pi}{2}$. For a plane wave, the electric and magnetic fields are contained in planes perpendicular to the direction of propagation $\vec{\gamma}$. The directions of polarization

$$
\overrightarrow{u_{\theta}}=\frac{\frac{d \vec{\gamma}}{d \theta}}{\left|\frac{d \vec{\gamma}}{d \theta}\right|}=\left(\begin{array}{c}
-\sin \theta \\
\cos \theta \\
0
\end{array}\right) \quad \text { and } \quad \overrightarrow{u_{\phi}}=\frac{\frac{d \vec{\gamma}}{d \phi}}{\left|\frac{d \vec{\gamma}}{d \phi}\right|}=\left(\begin{array}{c}
\cos \theta \cos \phi \\
\sin \theta \cos \phi \\
-\sin \phi
\end{array}\right)
$$


define these planes. The electric and magnetic fields must be linear combinations of these two vectors. We call a field $\theta$-polarized or $\phi$-polarized if it is solely in the $\overrightarrow{u_{\theta}}$ or $\overrightarrow{u_{\phi}}$ direction, respectively. An incident plane wave satisfies

$$
\mathbf{E}_{i n c}(x, y, z)=e^{i k_{e x t}\left(\gamma_{1} x+\gamma_{2} y+\gamma_{3} z\right)}\left[a \overrightarrow{u_{\theta}}+b \overrightarrow{u_{\phi}}\right]=e^{i k_{e x t} \vec{\gamma} \cdot \mathbf{x}}\left[a \overrightarrow{u_{\theta}}+b \overrightarrow{u_{\phi}}\right]
$$

for constants $a$ and $b$. The corresponding magnetic field is

$$
\mathbf{H}_{i n c}(x, y, z)=e^{i k_{e x t} \vec{\gamma} \cdot \mathbf{x}}\left[b \overrightarrow{u_{\theta}}-a \overrightarrow{u_{\phi}}\right] .
$$

Often, we are interested in normally incident plane waves. A normally incident $\phi$ polarized electric field with a $\theta$-polarized magnetic field have the form

$$
\mathbf{E}=e^{i k_{e x t} \vec{\gamma} \cdot \mathbf{x}} \overrightarrow{u_{\phi}}=\left(\begin{array}{c}
e^{i k_{e x t} z} \\
0 \\
0
\end{array}\right) \quad \mathbf{H}=e^{i k_{e x t} \vec{\gamma} \cdot \mathbf{x}} \overrightarrow{u_{\theta}}=\left(\begin{array}{c}
0 \\
e^{i k_{e x t} z} \\
0
\end{array}\right) .
$$

2.4. Pseudoperiodicity of the fields. Our incident fields are not quite periodic. Since $\epsilon_{e x t}=\mu_{e x t}=1$, the products $k_{e x t} \gamma_{1}$ and $k_{e x t} \gamma_{2}$ are real valued and can be written as

$$
k_{e x t} \gamma_{1}=\hat{m}+\alpha \quad \text { and } \quad k_{e x t} \gamma_{2}=\hat{n}+\beta
$$

for some integers $\hat{m}$ and $\hat{n}$ and some constants $\alpha, \beta \in\left(-\frac{1}{2}, \frac{1}{2}\right]$. The constants $\alpha$ and $\beta$ depend on $k_{\text {ext }}$ and on the angles of incidence $\phi$ and $\theta$. Since $\vec{\gamma}$ is a unit vector, we can write $\gamma_{3}$ in terms of $\alpha$ and $\beta$

$$
\gamma_{3}=\frac{\sqrt{k_{e x t}^{2}-(\hat{m}+\alpha)^{2}-(\hat{n}+\beta)^{2}}}{k_{e x t}} .
$$

Our electric field becomes

$$
\mathbf{E}_{i n c}(x, y, z)=e^{i(\alpha x+\beta y)} e^{i \hat{m} x+i \hat{n} y+i \sqrt{k_{e x t}^{2}-(\hat{m}+\alpha)^{2}-(\hat{n}+\beta)^{2}} z}[a \vec{\theta}+b \vec{\phi}] .
$$

The function

$$
e^{i(\alpha x+\beta y)}
$$

is not necessarily $2 \pi$ periodic in $x$ and $y$. We will call it a phasor function. The incident fields therefore are products of a phasor function and a doubly periodic function, and we call them pseudoperiodic.

For normally incident fields, the constants $\alpha$ and $\beta$ are both zero, and the fields are periodic and not merely pseudoperiodic.

2.5. Transmission. A quantity of interest in scattering problems is the transmission coefficient $T$. This is a comparison of the energy in the incident fields to that in the total external fields far from the scatterer. The Poynting vector

$$
\mathbf{S}=\overline{\mathbf{E}} \times \mathbf{H},
$$

with $\overline{\mathbf{E}}$ the complex conjugate of $\mathbf{E}$, is a measurement of an electromagnetic field's energy flux [16]. For any surface $F$ far from the scatterer $\Omega$ and normal to the incident fields, we define the incident and transmitted energy flows by

$$
U_{i n c}=\int_{F} \operatorname{Re}\left(\mathbf{S}_{i n c}(\mathbf{x}) \cdot \mathbf{n}(\mathbf{x})\right) d S(\mathbf{x}) \quad U_{t r a n}=\int_{F} \operatorname{Re}\left(\mathbf{S}_{e x t}(\mathbf{x}) \cdot \mathbf{n}(\mathbf{x})\right) d S(\mathbf{x}),
$$


where $\mathbf{n}$ is an outward unit normal to $F$. These flows are measures of the energy passing through the surface $F$. The transmission coefficient is defined as

$$
T=\sqrt{\frac{U_{t r a n}}{U_{\text {inc }}}} .
$$

In computing $T$, we take the surface $F$ to be far enough from $\Omega$ that the decaying modes of the field are negligible, so that only the propagating modes contribute to the integrals.

Of particular interest in scattering problems are frequencies near which the transmission coefficient changes dramatically. A sudden, sharp rise or fall in transmission is a behavior known to occur near resonant frequencies - frequencies where the linear system of integral equations is singular. Various resonances have been observed in photonic crystals, both theoretically and experimentally [11, 14, 12, 18, 29, 26]. Our periodic scatterers could model such crystals.

\section{The Green's functions}

3.1. A pseudoperiodic Green's function. A pseudoperiodic Green's function for this problem must satisfy

$$
\begin{aligned}
& \Delta G\left(\mathbf{x}^{\prime}-\mathbf{x}\right)+k^{2} G\left(\mathbf{x}^{\prime}-\mathbf{x}\right)= \\
& \quad-\delta\left(z^{\prime}-z\right) e^{i\left(\alpha\left(x^{\prime}-x\right)+\beta\left(y^{\prime}-y\right)\right)} \sum_{\mu, \nu} \delta\left(x^{\prime}-x+2 \pi \mu\right) \delta\left(y^{\prime}-y+2 \pi \nu\right)
\end{aligned}
$$

where the sums over $\mu$ and $\nu$ are from $-\infty$ to $\infty$ (we will use $\mu$ and $\nu$ for physical periodic reflections and $m$ and $n$ for Fourier modes). The values of $x$ and $y$ may fall within one period $(0 \leq x, y<2 \pi)$. The Green's function can be written as a Fourier series:

$$
G\left(\mathbf{x}^{\prime}-\mathbf{x}\right)=\frac{1}{8 \pi^{2}} \sum_{m, n} \frac{e^{-\sqrt{-\lambda_{m n}}\left|z^{\prime}-z\right|}}{\sqrt{-\lambda_{m n}}} P_{m n}
$$

where $P_{m n}=e^{i\left[(m+\alpha)\left(x^{\prime}-x\right)+(n+\beta)\left(y^{\prime}-y\right)\right]}$ and $\lambda_{m n}=k^{2}-(m+\alpha)^{2}-(n+\beta)^{2}$.

3.2. Properties of the Green's function. Although it is not clear from its appearance, this periodic Green's function has the same $1 / 4 \pi r$ singularity as the free space Helmholtz Green's function. The singularity in this function will be explored more in Sec. 6.

The constant $\lambda_{m n}=k^{2}-(m+\alpha)^{2}-(n+\beta)^{2}$ is important and determines the nature of each Fourier mode in the Green's function. In general, $k^{2}=\omega^{2} \epsilon \mu$ is a complex number with positive imaginary part. For $z$ away from 0 , each mode of the Green's function (3.2) is analytic in $\lambda_{m n}$ for all $\lambda_{m n}$ in the upper half complex plane except for $\lambda_{m n}=0$. Since $\lambda_{m n}$ never has a negative imaginary part, we can take the negative real axis as a branch cut for the square root function. We must use the same sign conventions with $\sqrt{-\lambda_{m n}}$ as in Sec. 2.2 in order to ensure proper decay and propagation of the modes.

Much of the physical interest in scattering deals with lossless media where the $\lambda_{m n}$ are real-valued. In this case, there are a finite number of modes (including $m=n=0$ ) for which $\sqrt{-\lambda_{m n}}$ is purely imaginary. These modes are known as oscillating or propagating modes [31]. They occur for the finite number of integers 
$m$ and $n$ such that $m^{2}+n^{2}<k^{2}$. The infinite number of other modes all decay as $z \rightarrow \pm \infty$ and are known as decaying modes.

At certain frequencies, it is possible that $\lambda_{m n}=0$ for one mode, in which case there is a singularity in the Green's function (3.2). At such modes, the $m, n$ term of the summation takes another form with a linear term. Wood's anomalies are a class of transmission resonances that occur near these singular frequencies.

\section{The Integral equations}

The scattering problem can be rewritten as a system of integral equations on the boundary $\partial \Omega$, known as the Müller integral equations. These are a set of electromagnetic integral equations which are Fredholm of the 2nd kind.

Müller derives the equations in [20] for a compact scatterer with the free-space Helmholtz Green's function. Since the singularities in the free-space and the periodic Green's functions are of the same nature, most of this derivation holds for our periodic scatterer. We outline the derivation here, supplying the details wherever they differ from the free-space case of [20].

Throughout this section, we will assume $\mathbf{E}$ and $\mathbf{H}$ are $C^{1}$. Whenever $\mathbf{E}$ or $\mathbf{H}$ appears without an argument in a surface integral, it will go unstated that the field is evaluated at $\mathbf{x}$. Whenever $G$ appears without an argument, it will go unstated that $G$ is evaluated at $\left(\mathbf{x}^{\prime}-\mathbf{x}\right)$.

4.1. Representation theorems. We begin with two representation theorems. These were originally derived for a free-space Green's function and a compact scatterer in [30], and the proofs can be found there and in [20]. In these theorems, $\mathbf{x}^{\prime}$ is not on the boundary $\partial \Omega$, and there are therefore no singularities in the integrals.

Theorem 4.1 (Interior Representation Theorem). Suppose $\mathbf{E}$ and $\mathbf{H}$ are pseudoperiodic solutions of Maxwell's equations in the interior of our scatterer $\Omega$. Then for $\mathbf{x}^{\prime}$ in the interior of $\Omega$

$$
\begin{aligned}
& \mathbf{E}\left(\mathbf{x}^{\prime}\right)=\int_{\partial \Omega}[-i \omega \mu(\mathbf{n} \times \mathbf{H}) G-(\mathbf{n} \times \mathbf{E}) \times \nabla G-(\mathbf{n} \cdot \mathbf{E}) \nabla G] d S(\mathbf{x}), \\
& \mathbf{H}\left(\mathbf{x}^{\prime}\right)=\int_{\partial \Omega}[i \omega \epsilon(\mathbf{n} \times \mathbf{E}) G-(\mathbf{n} \times \mathbf{H}) \times \nabla G-(\mathbf{n} \cdot \mathbf{H}) \nabla G] d S(\mathbf{x}) .
\end{aligned}
$$

Here $\mathbf{n}$ is the outward unit normal vector at $\mathbf{x}$. For $\mathbf{x}^{\prime}$ in the exterior of $\Omega$, both integrals above are 0.

The adaptation of the proof of this theorem to our periodic geometry is straightforward, since the singularities in the Green's function are the same. It is provided in [3].

We will now state and prove an exterior analogue to the interior representation theorem. The proof of this theorem differs from that of the non-periodic scatterer, and it merits inclusion. It was first proved in [21].

Theorem 4.2 (Exterior Representation Theorem). Suppose $\mathbf{E}$ and $\mathbf{H}$ are pseudoperiodic solutions of Maxwell's equations in the exterior of our scatterer $\Omega$. Suppose also that the radiation conditions (2.3) hold. Then for $\mathbf{x}^{\prime}$ in the exterior of $\Omega$

$$
\begin{aligned}
\mathbf{E}\left(\mathbf{x}^{\prime}\right) & =\int_{\partial \Omega}[i \omega \mu(\mathbf{n} \times \mathbf{H}) G+(\mathbf{n} \times \mathbf{E}) \times \nabla G+(\mathbf{n} \cdot \mathbf{E}) \nabla G] d S(\mathbf{x}), \\
\mathbf{H}\left(\mathbf{x}^{\prime}\right) & =\int_{\partial \Omega}[-i \omega \epsilon(\mathbf{n} \times \mathbf{E}) G+(\mathbf{n} \times \mathbf{H}) \times \nabla G+(\mathbf{n} \cdot \mathbf{H}) \nabla G] d S(\mathbf{x}) .
\end{aligned}
$$


For $\mathbf{x}^{\prime}$ in the interior of $\Omega$, both integrals above equal 0 .

Proof. We let $\mathbf{x}^{\prime}=\left(x^{\prime}, y^{\prime}, z^{\prime}\right)$. Let $S$ be the square cylinder given by one periodic block of space, $S=\{\mathbf{x}: 0 \leq x, y \leq 2 \pi\}$. Choose $M$ large enough that the planes $z= \pm M$ bound $\Omega$ and $\left|z^{\prime}\right|<M$. Let $\Omega_{M}$ be the set of points in the exterior of $\Omega$ such that $|z| \leq M$. This region is bounded by $\partial \Omega,|z|=M$, and $\partial S$. Now, apply the Interior Representation Thm. 4.1 to the region $\Omega_{M}$. We have, if $\mathbf{x}^{\prime} \in \Omega_{M}$,

$$
\begin{aligned}
\mathbf{E}\left(\mathbf{x}^{\prime}\right) & =\int_{\partial \Omega_{M}}[-i \omega \mu(\tilde{\mathbf{n}} \times \mathbf{H}) G-(\tilde{\mathbf{n}} \times \mathbf{E}) \times \nabla G-(\tilde{\mathbf{n}} \cdot \mathbf{E}) \nabla G] d S(\mathbf{x}), \\
\mathbf{H}\left(\mathbf{x}^{\prime}\right) & =\int_{\partial \Omega_{M}}[i \omega \epsilon(\tilde{\mathbf{n}} \times \mathbf{E}) G-(\tilde{\mathbf{n}} \times \mathbf{H}) \times \nabla G-(\tilde{\mathbf{n}} \cdot \mathbf{H}) \nabla G] d S(\mathbf{x}) .
\end{aligned}
$$

The vector $\tilde{\mathbf{n}}$ is the outward unit normal to $\partial \Omega_{M}$. If $\mathbf{x}^{\prime} \in \Omega$, by Thm. 4.1 both integrals in (4.1) are zero. We will break the integrals (4.1) into three boundary integrals. We will proceed here only with the $\mathbf{E}$ integral. The proof for the $\mathbf{H}$ integral is identical.

$$
\begin{aligned}
\mathbf{E}\left(\mathbf{x}^{\prime}\right)= & \int_{\partial \Omega}[i \omega \mu(\mathbf{n} \times \mathbf{H}) G+(\mathbf{n} \times \mathbf{E}) \times \nabla G+(\mathbf{n} \cdot \mathbf{E}) \nabla G] d S(\mathbf{x}) \\
& +\int_{\partial S \cap \partial \Omega_{M}}[-i \omega \mu(\tilde{\mathbf{n}} \times \mathbf{H}) G-(\tilde{\mathbf{n}} \times \mathbf{E}) \times \nabla G-(\tilde{\mathbf{n}} \cdot \mathbf{E}) \nabla G] d S(\mathbf{x}) \\
& +\int_{|z|=M}[-i \omega \mu(\tilde{\mathbf{n}} \times \mathbf{H}) G-(\tilde{\mathbf{n}} \times \mathbf{E}) \times \nabla G-(\tilde{\mathbf{n}} \cdot \mathbf{E}) \nabla G] d S(\mathbf{x}) .
\end{aligned}
$$

We use $\mathbf{n}$ to denote the outward unit normal on $\partial \Omega$, which is opposite in direction to the outward normal $\tilde{\mathbf{n}}$ of $\partial \Omega_{M}$. This accounts for the change in sign in the $\partial \Omega$ integral above.

We will show that the second of these integrals (4.2) is zero. The pseudoperiodic part of $\mathbf{E}$ is $e^{i \alpha x+i \beta y}$, while that of the Green's function (or its derivatives) is $e^{i \alpha\left(x^{\prime}-x\right)+i \beta\left(y^{\prime}-y\right)}$. In a product of $\mathbf{E}$ with $G$ (or a derivative of $G$ ), the $x$ and $y$ dependence of these phasors will cancel out. The integrand is therefore periodic. On opposite sides of $\partial S$, the $\partial S \cap \partial \Omega_{M}$ integrals cancel due to this periodicity and the fact that the normal vectors on opposite sides of $\partial S$ point in opposite directions. The second integral of (4.2) is therefore zero.

We will show that the integral over $z=M$ of (4.2) approaches 0 as $M \rightarrow \infty$. The $z=-M$ integral can be shown to approach 0 using the same reasoning. In the integrand, we substitute $i \omega \mu(\tilde{\mathbf{n}} \times \mathbf{H})=\tilde{\mathbf{n}} \times(\nabla \times \mathbf{E})$ from Maxwell's equations:

$$
\int_{z=M}[-\tilde{\mathbf{n}} \times(\nabla \times \mathbf{E}) G-(\tilde{\mathbf{n}} \times \mathbf{E}) \times \nabla G-(\tilde{\mathbf{n}} \cdot \mathbf{E}) \nabla G] d S(\mathbf{x}) .
$$

Here $\tilde{\mathbf{n}}=(0,0,1)$, and we expand the integrand:

$$
\left[\begin{array}{c}
G E_{1, z}-E_{1} G_{z}-G E_{3, x}-E_{3} G_{x} \\
G E_{2, z}-E_{2} G_{z}-G E_{3, y}-E_{3} G_{y} \\
E_{1} G_{x}+E_{2} G_{y}-E_{3} G_{z}
\end{array}\right]
$$

where $E_{1}, E_{2}$, and $E_{3}$ denote the $x, y$, and $z$ components of $\mathbf{E}$, respectively. We will first deal with the term $\left(G E_{1, z}-E_{1} G_{z}\right)$. As $M \rightarrow \infty$, we can represent the fields by 
the radiation conditions (2.3). With (3.2) for $G$, we can write

$$
\begin{aligned}
& \int_{z=M}\left(G\left(\mathbf{x}^{\prime}-\mathbf{x}\right) E_{1, z}(\mathbf{x})-E_{1}(\mathbf{x}) G_{z}\left(\mathbf{x}^{\prime}-\mathbf{x}\right)\right) d S(\mathbf{x}) \\
& =\frac{1}{8 \pi^{2}} \int_{0}^{2 \pi} \int_{0}^{2 \pi}\left(\sum_{m, n} e^{i(m+\alpha)\left(x^{\prime}-x\right)+i(n+\beta)\left(y^{\prime}-y\right)} \frac{e^{-\sqrt{-\lambda_{m n}}\left|z^{\prime}-M\right|}}{\sqrt{-\lambda_{m n}}}\right) \\
& \times\left(\sum_{m^{\prime}, n^{\prime}} E_{1, m^{\prime} n^{\prime}}^{+} e^{i\left(m^{\prime}+\alpha\right) x+i\left(n^{\prime}+\beta\right) y} \sqrt{-\lambda_{m^{\prime} n^{\prime}}} e^{-\sqrt{-\lambda_{m^{\prime} n^{\prime}}} M}\right) d x d y \\
& -\frac{1}{8 \pi^{2}} \int_{0}^{2 \pi} \int_{0}^{2 \pi}\left(\sum_{m, n} e^{i(m+\alpha)\left(x^{\prime}-x\right)+i(n+\beta)\left(y^{\prime}-y\right)} e^{-\sqrt{-\lambda_{m n}}\left|z^{\prime}-M\right|}\right) \\
& \quad \times\left(\sum_{m^{\prime}, n^{\prime}} E_{1, m^{\prime} n^{\prime}}^{+} e^{i\left(m^{\prime}+\alpha\right) x+i\left(n^{\prime}+\beta\right) y} e^{-\sqrt{-\lambda_{m^{\prime} n^{\prime}}} M}\right) d x d y .
\end{aligned}
$$

Each term in either integrand of (4.4) is of the form

$$
\int_{0}^{2 \pi} \int_{0}^{2 \pi} C e^{i(m+\alpha)\left(x^{\prime}-x\right)+i(n+\beta)\left(y^{\prime}-y\right)} e^{i\left(m^{\prime}+\alpha\right) x+i\left(n^{\prime}+\beta\right) y} d x d y
$$

for some constant $C$. Rearranging and canceling $\alpha$ and $\beta$, we see that most of these terms are 0 :

$$
\begin{aligned}
& \int_{0}^{2 \pi} \int_{0}^{2 \pi} C e^{i(m+\alpha) x^{\prime}+i(n+\beta) y^{\prime}} e^{i\left(m^{\prime}-m\right) x+i\left(n^{\prime}-n\right) y} d x d y \\
= & 4 \pi^{2} C e^{i(m+\alpha) x^{\prime}+i(n+\beta) y^{\prime}} \delta_{m-m^{\prime}} \delta_{n-n^{\prime}} .
\end{aligned}
$$

The non-zero terms of the integrands in (4.4) are those for which $m^{\prime}=m$ and $n^{\prime}=n$, and (4.4) reduces to

$$
\begin{aligned}
& \int_{z=M}\left(G\left(\mathbf{x}^{\prime}-\mathbf{x}\right) E_{1, z}(\mathbf{x})-E_{1}(\mathbf{x}) G_{z}\left(\mathbf{x}^{\prime}-\mathbf{x}\right)\right) d S(\mathbf{x}) \\
= & \frac{e^{i(m+\alpha) x^{\prime}+i(n+\beta) y^{\prime}}}{8 \pi^{2}} \int_{0}^{2 \pi} \int_{0}^{2 \pi}\left(\sum_{m, n} \frac{e^{-\sqrt{-\lambda_{m n}}\left|z^{\prime}-M\right|}}{\sqrt{-\lambda_{m n}}} E_{1, m n}^{+} \sqrt{-\lambda_{m n}} e^{-\sqrt{-\lambda_{m n}} M}\right) d x d y \\
& -\frac{e^{i(m+\alpha) x^{\prime}+i(n+\beta) y^{\prime}}}{8 \pi^{2}} \int_{0}^{2 \pi} \int_{0}^{2 \pi}\left(\sum_{m, n} e^{-\sqrt{-\lambda_{m n}}\left|z^{\prime}-M\right|} E_{1, m n}^{+} e^{-\sqrt{-\lambda_{m n}} M}\right) d x d y .
\end{aligned}
$$

which clearly sums to 0 .

The terms $G E_{2, z}-E_{2} G_{z},-G E_{3, x}-E_{3} G_{x}$, and $-G E_{3, y}-E_{3} G_{y}$ in (4.3) can all be shown in the same way to integrate to 0 as $M \rightarrow \infty$. There is a sign change from the derivative of the Green's function that makes this possible. We are left with the term $E_{1} G_{x}+E_{2} G_{y}-E_{3} G_{z}$, which we first integrate by parts:

$$
\int_{z=M} E_{1} G_{x}+E_{2} G_{y}-E_{3} G_{z} d x d y=\int_{z=M}-E_{1, x} G-E_{2, y} G+E_{3} G_{z} d x d y .
$$

The boundary from the integration by parts is zero due to the periodicity of the integrand. Now, since $\mathbf{E}$ is divergence free, $-E_{1, x} G-E_{2, y} G=-E_{3, z} G$, and (4.6) 
becomes

$$
\int_{z=M} E_{3} G_{z}-E_{3, z} G d x d y
$$

This integral can be shown to approach 0 in the same way that the integral (4.4) does.

The other surface integrals of (4.2) have been shown to be zero as $M \rightarrow \infty$, and we are left with our result for $\mathbf{E}$

$$
\mathbf{E}\left(\mathbf{x}^{\prime}\right)=\int_{\partial \Omega}[i \omega \mu(\mathbf{n} \times \mathbf{H}) G+(\mathbf{n} \times \mathbf{E}) \times \nabla G+(\mathbf{n} \cdot \mathbf{E}) \nabla G] d S(\mathbf{x}) .
$$

As stated, the proof for $\mathbf{H}$ follows the same steps.

4.2. Surface currents and charges. We do not work directly with $\mathbf{E}$ and $\mathbf{H}$ on the surface $\partial \Omega$. The integral equations are instead in terms of tangential components of the fields known as surface currents $\mathbf{j}$ and $\mathbf{j}^{\prime}$ :

$$
\mathbf{j}=-\mathbf{n} \times \mathbf{H} \quad \mathbf{j}^{\prime}=\mathbf{n} \times \mathbf{E} .
$$

These surface currents are also known as the traces of the fields on $\partial \Omega$. We also define the surface charges $\rho$ and $\rho^{\prime}$.

$$
i \omega \rho=\nabla_{s} \cdot \mathbf{j} \quad i \omega \rho^{\prime}=\nabla_{s} \cdot \mathbf{j}^{\prime} .
$$

Here $\nabla_{s} \cdot$ is the surface divergence as defined in [20, page 157].

We will restate Thms. 4.1 and 4.2 in terms of $\mathbf{j}$ and $\mathbf{j}^{\prime}$. To do this, we need the relations

$$
\mathbf{n} \cdot \mathbf{E}=-\rho / \epsilon \quad \mathbf{n} \cdot \mathbf{H}=-\rho^{\prime} / \epsilon
$$

which are proved in [20, page 159].

The integrals of the interior representation theorem become:

$$
\begin{aligned}
\mathbf{E}\left(\mathbf{x}^{\prime}\right) & =\int_{\partial \Omega}\left[i \omega \mu \mathbf{j}(\mathbf{x}) G-\mathbf{j}^{\prime}(\mathbf{x}) \times \nabla G+\frac{\rho(\mathbf{x})}{\epsilon} \nabla G\right] d S(\mathbf{x}), \\
\mathbf{H}\left(\mathbf{x}^{\prime}\right) & =\int_{\partial \Omega}\left[i \omega \epsilon \mathbf{j}^{\prime}(\mathbf{x}) G+\mathbf{j}(\mathbf{x}) \times \nabla G+\frac{\rho^{\prime}(\mathbf{x})}{\mu} \nabla G\right] d S(\mathbf{x}),
\end{aligned}
$$

and the integrals of the exterior representation theorem are

$$
\begin{aligned}
\mathbf{E}\left(\mathbf{x}^{\prime}\right) & =\int_{\partial \Omega}\left[-i \omega \mu \mathbf{j}(\mathbf{x}) G+\mathbf{j}^{\prime}(\mathbf{x}) \times \nabla G-\frac{\rho(\mathbf{x})}{\epsilon} \nabla G\right] d S(\mathbf{x}), \\
\mathbf{H}\left(\mathbf{x}^{\prime}\right) & =\int_{\partial \Omega}\left[-i \omega \epsilon \mathbf{j}^{\prime}(\mathbf{x}) G-\mathbf{j}(\mathbf{x}) \times \nabla G-\frac{\rho^{\prime}(\mathbf{x})}{\mu} \nabla G\right] d S(\mathbf{x}) .
\end{aligned}
$$

4.3. Jump conditions. We will investigate the limits of various integrals as $\mathbf{x}^{\prime}$ approaches $\partial \Omega$ from the interior and exterior along a normal direction. The results of this section depend heavily on the singularity of the Green's function. Since the singularities in the periodic problem are the same as those in the non-periodic problem, we will not include the proofs here and will instead refer the reader to the proofs in [20, pages 202-205]. 
Proposition 4.3. For a tangential surface vector $\mathbf{j}$ continuous on $\partial \Omega$, the integral

$$
\int_{\partial \Omega} \mathbf{j}(\mathbf{x}) G\left(\mathbf{x}^{\prime}-\mathbf{x}\right) d S(\mathbf{x})
$$

is continuous for all $\mathbf{x}^{\prime}$.

We will adopt the notation of [20] and define

$$
\int_{i n t \rightarrow \partial \Omega} G\left(\mathbf{x}^{\prime}-\mathbf{x}\right) d S(\mathbf{x})=\lim _{\mathbf{y} \rightarrow \mathbf{x}^{\prime}} \int_{\partial \Omega} G(\mathbf{y}-\mathbf{x}) d S(\mathbf{x})
$$

where the limit on the right hand side is taken as $\mathbf{y} \rightarrow \mathbf{x}^{\prime}$ for $\mathbf{y}$ in the interior of $\Omega$ along a direction normal to $\partial \Omega$ at $\mathbf{x}^{\prime} \in \partial \Omega$. We define $\int_{e x t \rightarrow \partial \Omega}$ similarly for $\mathbf{y}$ along an exterior normal.

Proposition 4.4. For our surface charges $\rho$ as defined, and for all $\mathbf{x}^{\prime} \in \partial \Omega$, the integral

$$
\int_{\partial \Omega} \mathbf{n}\left(\mathbf{x}^{\prime}\right) \times \rho(\mathbf{x}) \nabla G\left(\mathbf{x}^{\prime}-\mathbf{x}\right) d S(\mathbf{x})
$$

is well-defined as a principal value integral, and

$\mathbf{n}\left(\mathbf{x}^{\prime}\right) \times \int_{i n t \rightarrow \partial \Omega} \rho(\mathbf{x}) \nabla G\left(\mathbf{x}^{\prime}-\mathbf{x}\right) d S(\mathbf{x})=\mathbf{n}\left(\mathbf{x}^{\prime}\right) \times \int_{e x t \rightarrow \partial \Omega} \rho(\mathbf{x}) \nabla G\left(\mathbf{x}^{\prime}-\mathbf{x}\right) d S(\mathbf{x})$.

We will use the integral (4.10) to denote either of the limits of (4.11).

Proposition 4.5. For $\mathbf{x}^{\prime} \in \partial \Omega$ and $\mathbf{j}$ a continuous surface vector field on $\partial \Omega$, the integral

$$
\int_{\partial \Omega} \mathbf{n}\left(\mathbf{x}^{\prime}\right) \times[\mathbf{j}(\mathbf{x}) \times \nabla G] d S(\mathbf{x})
$$

is absolutely integrable, and

$$
\begin{aligned}
& \mathbf{n}\left(\mathbf{x}^{\prime}\right) \times \int_{i n t \rightarrow \partial \Omega} \mathbf{j}(\mathbf{x}) \times \nabla G d S(\mathbf{x})=-\frac{1}{2} \mathbf{j}\left(\mathbf{x}^{\prime}\right)+\int_{\partial \Omega} \mathbf{n}\left(\mathbf{x}^{\prime}\right) \times[\mathbf{j}(\mathbf{x}) \times \nabla G] d S(\mathbf{x}), \\
& \mathbf{n}\left(\mathbf{x}^{\prime}\right) \times \int_{e x t \rightarrow \partial \Omega} \mathbf{j}(\mathbf{x}) \times \nabla G d S(\mathbf{x})=+\frac{1}{2} \mathbf{j}\left(\mathbf{x}^{\prime}\right)+\int_{\partial \Omega} \mathbf{n}\left(\mathbf{x}^{\prime}\right) \times[\mathbf{j}(\mathbf{x}) \times \nabla G] d S(\mathbf{x}) .
\end{aligned}
$$

4.4. The field equations. We will apply the results of Sec. 4.3 to the interior and exterior representation theorems for the interior fields $\mathbf{E}_{\text {int }}$ and $\mathbf{H}_{\text {int }}$, the scattered fields $\mathbf{E}_{\text {scatt }}$ and $\mathbf{H}_{\text {scatt }}$, and the incident fields $\mathbf{E}_{\text {inc }}$ and $\mathbf{H}_{\text {inc }}$.

4.4.1. Interior fields. Let $\mathbf{y}$ lie in the interior of $\Omega$, and let $\mathbf{x}^{\prime} \in \partial \Omega$. We take the cross product of $\mathbf{n}\left(\mathbf{x}^{\prime}\right)$ with each side of the equations of the interior representation Thm. (4.8). We then take the limit as $\mathbf{y} \rightarrow \mathbf{x}^{\prime}$ along the interior normal. Then, using 
all of the propositions of Sec. 4.3 and combining the $\mathbf{j}\left(\mathbf{x}^{\prime}\right)$ terms, we have

$$
\begin{aligned}
\frac{1}{2} \mathbf{j}^{\prime}{ }_{i n t}\left(\mathbf{x}^{\prime}\right)= & \int_{\partial \Omega} i \omega \mu_{i n t}\left[\mathbf{n}\left(\mathbf{x}^{\prime}\right) \times \mathbf{j}_{i n t}(\mathbf{x})\right] G^{i n t} d S(\mathbf{x}) \\
& -\int_{\partial \Omega} \mathbf{n}\left(\mathbf{x}^{\prime}\right) \times\left[\mathbf{j}^{\prime}{ }_{i n t}(\mathbf{x}) \times \nabla G^{i n t}\right] d S(\mathbf{x}) \\
& +\frac{1}{\epsilon_{i n t}} \int_{\partial \Omega} \mathbf{n}\left(\mathbf{x}^{\prime}\right) \times \rho_{\text {int }}(\mathbf{x}) \nabla G^{i n t} d S(\mathbf{x}) \\
-\frac{1}{2} \mathbf{j}_{i n t}\left(\mathbf{x}^{\prime}\right)= & \int_{\partial \Omega} i \omega \epsilon_{i n t}\left[\mathbf{n}\left(\mathbf{x}^{\prime}\right) \times \mathbf{j}_{i n t}^{\prime}(\mathbf{x})\right] G^{i n t} d S(\mathbf{x}) \\
& +\int_{\partial \Omega} \mathbf{n}\left(\mathbf{x}^{\prime}\right) \times\left[\mathbf{j}_{\text {int }}(\mathbf{x}) \times \nabla G^{i n t}\right] d S(\mathbf{x}) \\
& +\frac{1}{\mu_{i n t}} \int_{\partial \Omega} \mathbf{n}\left(\mathbf{x}^{\prime}\right) \times \rho_{i n t}^{\prime}(\mathbf{x}) \nabla G^{i n t} d S(\mathbf{x}) .
\end{aligned}
$$

4.4.2. Scattered fields. Let $\mathbf{y}$ lie in the exterior of $\Omega$, and let $\mathbf{x}^{\prime} \in \partial \Omega$. We take the cross product of $\mathbf{n}\left(\mathbf{x}^{\prime}\right)$ with each side of the equations of the exterior representation Thm. (4.9) for the scattered fields. We then take the limit as $\mathbf{y} \rightarrow \mathbf{x}^{\prime}$ along the exterior normal. Then, the propositions of Sec. 4.3 give us

$$
\begin{aligned}
\frac{1}{2} \mathbf{j}_{\text {scatt }}\left(\mathbf{x}^{\prime}\right)= & -\int_{\partial \Omega} i \omega \mu_{\text {ext }}\left[\mathbf{n}\left(\mathbf{x}^{\prime}\right) \times \mathbf{j}_{\text {scatt }}(\mathbf{x})\right] G^{e x t} d S(\mathbf{x}) \\
& +\int_{\partial \Omega} \mathbf{n}\left(\mathbf{x}^{\prime}\right) \times\left[\mathbf{j}_{\text {scatt }}^{\prime}(\mathbf{x}) \times \nabla G^{e x t}\right] d S(\mathbf{x}) \\
& -\frac{1}{\epsilon_{\text {ext }}} \int_{\partial \Omega} \mathbf{n}\left(\mathbf{x}^{\prime}\right) \times \rho_{\text {scatt }}(\mathbf{x}) \nabla G^{e x t} d S(\mathbf{x}) \\
-\frac{1}{2} \mathbf{j}_{\text {scatt }}\left(\mathbf{x}^{\prime}\right)= & -\int_{\partial \Omega} i \omega \epsilon_{\text {ext }}\left[\mathbf{n}\left(\mathbf{x}^{\prime}\right) \times \mathbf{j}_{\text {scatt }}^{\prime}(\mathbf{x})\right] G^{e x t} d S(\mathbf{x}) \\
& -\int_{\partial \Omega} \mathbf{n}\left(\mathbf{x}^{\prime}\right) \times\left[\mathbf{j}_{\text {scatt }}(\mathbf{x}) \times \nabla G^{e x t}\right] d S(\mathbf{x}) \\
& -\frac{1}{\mu_{e x t}} \int_{\partial \Omega} \mathbf{n}\left(\mathbf{x}^{\prime}\right) \times \rho_{\text {scatt }}^{\prime}(\mathbf{x}) \nabla G^{e x t} d S(\mathbf{x})
\end{aligned}
$$

4.4.3. Incident fields. The incident fields are, by definition, the fields that would exist in the absence of the scatterer. In other words, they are the fields that would exist if $\epsilon=\epsilon_{e x t}$ and $\mu=\mu_{\text {ext }}$ everywhere. The interior representation formulas (4.8) are valid for the incident fields with these constants and with the Green's function $G^{e x t}$. We do not use the exterior representation theorem for the incident fields because this theorem depends on the radiation conditions, and the incident fields do not necessarily satisfy these conditions.

Let $\mathbf{y}$ lie in the interior of $\Omega$, and let $\mathbf{x}^{\prime} \in \partial \Omega$. Apply the interior representation Thm. (4.8) to the incident fields (but with the exterior constants and Green's function). We take the cross product of $\mathbf{n}\left(\mathbf{x}^{\prime}\right)$ with each side of the resulting equations. We then take the limit as $\mathbf{y} \rightarrow \mathbf{x}^{\prime}$ along the interior normal. Then, the propositions 
of Sec. 4.3 give us

$$
\begin{aligned}
\frac{1}{2} \mathbf{j}^{\prime}{ }_{i n c}\left(\mathbf{x}^{\prime}\right)= & \int_{\partial \Omega} i \omega \mu_{e x t}\left[\mathbf{n}\left(\mathbf{x}^{\prime}\right) \times \mathbf{j}_{i n c}(\mathbf{x})\right] G^{e x t} d S(\mathbf{x}) \\
& -\int_{\partial \Omega} \mathbf{n}\left(\mathbf{x}^{\prime}\right) \times\left[\mathbf{j}^{\prime}{ }_{i n c}(\mathbf{x}) \times \nabla G^{e x t}\right] d S(\mathbf{x}) \\
& +\frac{1}{\epsilon_{e x t}} \int_{\partial \Omega} \mathbf{n}\left(\mathbf{x}^{\prime}\right) \times \rho_{i n c}(\mathbf{x}) \nabla G^{e x t} d S(\mathbf{x}) \\
-\frac{1}{2} \mathbf{j}_{i n c}\left(\mathbf{x}^{\prime}\right)= & \int_{\partial \Omega} i \omega \epsilon_{e x t}\left[\mathbf{n}\left(\mathbf{x}^{\prime}\right) \times \mathbf{j}^{\prime}{ }_{i n c}(\mathbf{x})\right] G^{e x t} d S(\mathbf{x}) \\
& +\int_{\partial \Omega} \mathbf{n}\left(\mathbf{x}^{\prime}\right) \times\left[\mathbf{j}_{i n c}(\mathbf{x}) \times \nabla G^{e x t}\right] d S(\mathbf{x}) \\
& +\frac{1}{\mu_{e x t}} \int_{\partial \Omega} \mathbf{n}\left(\mathbf{x}^{\prime}\right) \times \rho_{i n c}^{\prime}(\mathbf{x}) \nabla G^{e x t} d S(\mathbf{x}) .
\end{aligned}
$$

4.4.4. Matching conditions. In terms of the surface currents, the boundary conditions of tangential continuity at $\partial \Omega$ are:

$$
\mathbf{j}_{\text {scatt }}=\mathbf{j}_{\text {int }}-\mathbf{j}_{\text {inc }} \quad \text { and } \quad \mathbf{j}_{\text {scatt }}^{\prime}=\mathbf{j}_{\text {int }}^{\prime}-\mathbf{j}_{\text {inc }}^{\prime} .
$$

We add Equ. (4.12) to (4.13) and substitute these boundary conditions for the scattered currents on both sides. Some of the resulting integrals (those involving $\mathbf{j}_{\text {inc }}$ ) are the same as the integrals in the incident Equs. (4.14). We can substitute the left hand side of (4.14) for these integrals, and we get the Müller equations. Before stating the equations, we make one change. Two of the integrals involve the surface divergence of a surface current. In order to make these integrals more manageable, we replace them as in the following proposition, which is proved in [20, page 300]:

Proposition 4.6. With all quantities as previously defined

$$
\begin{gathered}
\int_{\partial \Omega} \mathbf{n}\left(\mathbf{x}^{\prime}\right) \times \rho(\mathbf{x}) \nabla\left(G^{e x t}-G^{i n t}\right) d S(\mathbf{x}) \\
=\int_{\partial \Omega} \mathbf{n}\left(\mathbf{x}^{\prime}\right) \times\left[(\mathbf{j}(\mathbf{x}) \cdot \nabla) \nabla\left(G^{e x t}-G^{i n t}\right)\right] d S(\mathbf{x}) .
\end{gathered}
$$

4.5. The Müller integral equations. We rearrange a few constants and make the substitutions of Prop. 4.6 to obtain the Müller equations in their final form [20, page 319]:

$$
\begin{aligned}
\mathbf{j}_{i n c}\left(\mathbf{x}^{\prime}\right)= & \frac{\mu_{i n t}+\mu_{e x t}}{2 \mu_{e x t}} \mathbf{j}_{\text {int }}\left(\mathbf{x}^{\prime}\right) \\
& -\frac{1}{\mu_{e x t}} \int_{\partial \Omega} \mathbf{n}\left(\mathbf{x}^{\prime}\right) \times\left[\mathbf{j}_{\text {int }}(\mathbf{x}) \times \nabla\left(\mu_{e x t} G^{e x t}-\mu_{i n t} G^{i n t}\right)\right] d S(\mathbf{x}) \\
& -\frac{i}{\mu_{e x t} \omega} \int_{\partial \Omega}\left[\mathbf{n}\left(\mathbf{x}^{\prime}\right) \times \mathbf{j}_{\text {int }}^{\prime}(\mathbf{x})\right]\left(k_{e x t}^{2} G^{e x t}-k_{i n t}^{2} G^{i n t}\right) d S(\mathbf{x}) \\
& -\frac{i}{\mu_{e x t} \omega} \int_{\partial \Omega} \mathbf{n}\left(\mathbf{x}^{\prime}\right) \times\left[\left(\mathbf{j}_{i n t}^{\prime}(\mathbf{x}) \cdot \nabla\right) \nabla\left(G^{e x t}-G^{i n t}\right)\right] d S(\mathbf{x}), \\
\mathbf{j}_{\text {inc }}^{\prime}\left(\mathbf{x}^{\prime}\right)= & \frac{\epsilon_{i n t}+\epsilon_{\text {ext }}}{2 \epsilon_{\text {ext }}} \mathbf{j}_{\text {int }}^{\prime}\left(\mathbf{x}^{\prime}\right)
\end{aligned}
$$




$$
\begin{aligned}
& -\frac{1}{\epsilon_{e x t}} \int_{\partial \Omega} \mathbf{n}\left(\mathbf{x}^{\prime}\right) \times\left[\mathbf{j}_{\text {int }}^{\prime}(\mathbf{x}) \times \nabla\left(\epsilon_{\text {ext }} G^{\text {ext }}-\epsilon_{i n t} G^{i n t}\right)\right] d S(\mathbf{x}) \\
& -\frac{i}{\epsilon_{\text {ext }} \omega} \int_{\partial \Omega}\left[\mathbf{n}\left(\mathbf{x}^{\prime}\right) \times \mathbf{j}_{\text {int }}(\mathbf{x})\right]\left(k_{\text {ext }}^{2} G^{e x t}-k_{i n t}^{2} G^{i n t}\right) d S(\mathbf{x}) \\
& -\frac{i}{\epsilon_{\text {ext }} \omega} \int_{\partial \Omega} \mathbf{n}\left(\mathbf{x}^{\prime}\right) \times\left[\left(\mathbf{j}_{\text {int }}(\mathbf{x}) \cdot \nabla\right) \nabla\left(G^{e x t}-G^{i n t}\right)\right] d S(\mathbf{x}) .
\end{aligned}
$$

The Müller equations are a coupled system of integral equations. The incident currents $\mathbf{j}_{\text {inc }}$ and $\mathbf{j}_{\text {inc }}^{\prime}$ are known. This system of integral equations can be solved for the interior surface currents $\mathbf{j}_{\text {int }}$ and $\mathbf{j}_{\text {int }}^{\prime}$.

Once we have the interior surface currents, we can use the interior and exterior representation theorems to find the scattered and the interior electric and magnetic fields:

$$
\begin{aligned}
\mathbf{E}_{\text {int }}\left(\mathbf{x}^{\prime}\right) & =\int_{\partial \Omega}\left[i \omega \mu_{i n t} \mathbf{j}_{\text {int }}(\mathbf{x}) G^{i n t}-\mathbf{j}_{\text {int }}^{\prime}(\mathbf{x}) \times \nabla G^{i n t}+\frac{1}{\epsilon_{\text {int }}}(\mathbf{j}(\mathbf{x}) \cdot \nabla) \nabla G^{i n t}\right] d S(\mathbf{x}) \\
\mathbf{H}_{\text {int }}\left(\mathbf{x}^{\prime}\right) & =\int_{\partial \Omega}\left[i \omega \epsilon_{i n t} \mathbf{j}_{\text {int }}^{\prime}(\mathbf{x}) G^{i n t}+\mathbf{j}_{\text {int }}(\mathbf{x}) \times \nabla G^{i n t}+\frac{1}{\mu_{i n t}}\left(\mathbf{j}^{\prime}(\mathbf{x}) \cdot \nabla\right) \nabla G^{i n t}\right] d S(\mathbf{x}) \\
\mathbf{E}_{\text {scatt }}\left(\mathbf{x}^{\prime}\right) & =-\int_{\partial \Omega}\left[i \omega \mu_{\text {ext }} \mathbf{j}_{\text {int }}(\mathbf{x}) G^{\text {ext }}-\mathbf{j}_{\text {int }}^{\prime}(\mathbf{x}) \times \nabla G^{\text {ext }}+\frac{1}{\epsilon_{\text {ext }}}(\mathbf{j}(\mathbf{x}) \cdot \nabla) \nabla G^{e x t}\right] d S(\mathbf{x}) \\
\mathbf{H}_{\text {scatt }}\left(\mathbf{x}^{\prime}\right) & =-\int_{\partial \Omega}\left[i \omega \epsilon_{\text {ext }} \mathbf{j}_{\text {int }}^{\prime}(\mathbf{x}) G^{\text {ext }}+\mathbf{j}_{\text {int }}(\mathbf{x}) \times \nabla G^{\text {ext }}+\frac{1}{\mu_{\text {ext }}}\left(\mathbf{j}^{\prime}(\mathbf{x}) \cdot \nabla\right) \nabla G^{e x t}\right] d S(\mathbf{x}) .
\end{aligned}
$$

4.6. Singularities in the integral equations. Since the Green's functions have singularities at $\mathbf{x}=\mathbf{0}$, the Müller equations include some convergent improper integrals. The integrals:

$$
\int_{\partial \Omega}\left[\mathbf{n}\left(\mathbf{x}^{\prime}\right) \times \mathbf{j}(\mathbf{x})\right]\left(k_{e x t}^{2} G^{e x t}-k_{i n t}^{2} G^{i n t}\right) d S(\mathbf{x})
$$

and

$$
\int_{\partial \Omega} \mathbf{n}\left(\mathbf{x}^{\prime}\right) \times\left[\mathbf{j}(\mathbf{x}) \times \nabla\left(\epsilon_{e x t} G^{e x t}-\epsilon_{i n t} G^{i n t}\right)\right] d S(\mathbf{x})
$$

are both singular integrals. The first of these, (4.17), is a single layer potential. The second, (4.18), contains something like a double layer potential as well as another type of singular integral. These will be investigated more in Sec. 6. The other integrals that appear in the Müller equations are not singular, as the singularities are subtracted out in the difference $G_{\text {ext }}-G_{\text {int }}$.

\section{Ewald splitting}

The numerical solution of the Müller Equs (4.15) will require many evaluations of the Green's function and its derivatives. There are two numerical difficulties associated with the Green's function (3.2). First, this function converges so slowly when $z$ is small that it unusable computationally. Second, the singularity in the Green's function is difficult to analyze and to work with. We will deal with the first problem in this section and with the second in Sec. 6 .

We use Ewald summation to accelerate computations of the Green's function. The method splits the Green's function into two parts which each converge more quickly than the Fourier series (3.2) representation. This method was first presented 
in [13]. Our 3D use of the technique mirrors the 2D approach of [17] and [23]. We write the Green's function in two parts:

$$
G\left(\mathbf{x}^{\prime}-\mathbf{x}\right)=G 1\left(\mathbf{x}^{\prime}-\mathbf{x}\right)+G 2\left(\mathbf{x}^{\prime}-\mathbf{x}\right),
$$

with

$$
\begin{aligned}
& G 1\left(\mathbf{x}^{\prime}-\mathbf{x}\right)=\frac{1}{8 \pi^{3 / 2}} \sum_{\mu, \nu} e^{-2 \pi i(\alpha \mu+\beta \nu)} \sum_{\ell=0}^{\infty} \frac{k^{2 \ell} E^{2 \ell-1}}{\ell !} \operatorname{ExpInt}_{\ell+1 / 2}\left(\frac{R_{\mu \nu}^{2}}{4 E^{2}}\right), \\
& G 2\left(\mathbf{x}^{\prime}-\mathbf{x}\right)=\frac{1}{8 \pi^{5 / 2}} \sum_{m, n} P_{m n} \sum_{\ell=0}^{\infty} \frac{(-1)^{\ell}\left(z^{\prime}-z\right)^{2 \ell}}{4^{\ell} \ell ! E^{2 \ell-1}} \operatorname{ExpInt}_{\ell+1 / 2}\left(-\lambda_{m n} E^{2}\right),
\end{aligned}
$$

where $E$ is a parameter that we are free to choose, known as the Ewald splitting parameter. Here, $\operatorname{ExpInt}_{n}$ are exponential integral equations of order $n$ (see Appendix A), $R_{\mu \nu}^{2}=\left(x^{\prime}-x-2 \pi \mu\right)^{2}+\left(y^{\prime}-y-2 \pi \nu\right)^{2}+\left(z-z^{\prime}\right)^{2}$, and $P_{m n}$ and $\lambda_{m n}$ are as previously defined. Note that $G 1$ consists of sums of periodic reflections while the $G 2$ sums are over Fourier modes. The singularity is entirely contained in $G 1$, and $G 2$ is smooth. If $E$ is chosen well, the sums in $G 1$ and $G 2$ have much better convergence properties than those in (3.2), since exponential integral functions of any order decay exponentially. Convergence in both $G 1$ and $G 2$ is better for small $k$.

There is a trade off in the selection of the splitting parameter $E$. If $E$ is large, $G 2$ converges quickly, but $G 1$ converges slowly. If $E$ is small, the opposite occurs. We have found it easy to experimentally determine a choice for $E$. More information can be found in [8] and [9].

Recall that in order to satisfy the radiation conditions (2.3), we choose the negative square root in (3.2) whenever $\operatorname{Re} \sqrt{-\lambda_{m n}}=0$. When evaluating $G 2$ we do this by choosing the negative square root in the evaluation of the exponential integral functions (see A).

\section{Smoothing and correction}

Two types of integrals that appear in the integral Equs. (4.15) are singular integrals. The singularities introduce errors in basic numerical integration techniques that are on the order of the step size. The other integrals in Müller's equations are smooth and pose no problems numerically. Our method for dealing with the singular integrals involves smoothing the integrand and then adding a correction term to the resulting integral. These types of methods were first developed by Beale in [4] for problems in periodic water waves. They have been furthered in [5] and [6].

The analysis of this section is for a general $C^{3}$ surface $\partial \Omega$. This condition allows us to take the necessary Taylor expansions of functions on the surface.

It is not immediately clear how to regularize the pseudoperiodic Green's function (3.2). Multiplying by a smoothing function $1-e^{-\frac{r^{2}}{\delta^{2}}}$ as in $[4,5,6]$ may remove the singularity, but it also destroys periodicity. It is not clear how to pick a smoothing function for this $G$ that is also periodic.

The Ewald formulation of the Green's function fortunately provides a clear way to achieve smoothing. The singularity in $G$ is entirely contained in the $G 1$ summation (5.1) $R_{\mu \nu} \rightarrow 0$. We use a slightly different representation of $G 1$, however, to develop a straightforward smoothing of the Green's function. Written in this way,

$$
G 1\left(\mathbf{x}^{\prime}-\mathbf{x}\right)=\frac{1}{8 \pi^{3 / 2}} \sum_{\mu, \nu} e^{-2 \pi i(\alpha \mu+\beta \nu)} \int_{0}^{E^{2}} \frac{e^{k^{2} t-\frac{R_{\mu \nu}^{2}}{4 t}}}{t^{3 / 2}} d t
$$


we can see that the singularity is located at the zero endpoint of integration of the $d t$ integral as $r=\left|\mathbf{x}^{\prime}-\mathbf{x}\right| \rightarrow 0$. If we just cut this singularity out by integrating from some small function of $\delta$ instead of from zero, we obtain a $G 1_{\delta}$ that is smooth:

$$
G 1_{\delta}\left(\mathbf{x}^{\prime}-\mathbf{x}\right)=\frac{1}{8 \pi^{3 / 2}} \sum_{\mu, \nu} e^{-2 \pi i(\alpha \mu+\beta \nu)} \int_{\delta^{2}}^{E^{2}} \frac{e^{k^{2} t-\frac{R_{\mu \nu}^{2}}{4 t}}}{t^{3 / 2}} d t
$$

This smoothed Green's function is not singular at $r=0$, and it quickly approaches $G 1$ as $r$ increases (see Fig. 6.1). It is also still periodic. Our strategy is therefore to replace $G$ with $G_{\delta}$ in any of the singular integrals that appear in the Müller integral equations (introducing a smoothing error), to evaluate these integrals using a trapezoid method (accurately, since the integrands are now smooth), and to add correction terms to compensate for the smoothing error introduced. The error introduced through discretization of the smoothed integrals will be discussed at the end of this section (see Sec. 6.1).
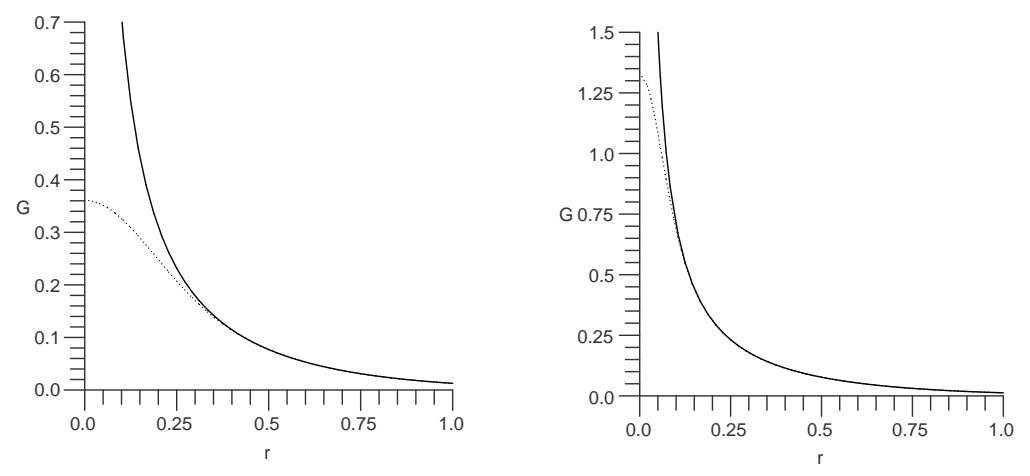

FIG. 6.1. $G 1$ and $G 1_{\delta}$ on the same axes. The left plot is with $\delta=0.01$. On the right, $\delta=.001$. Both are for normally incident light. $E=0.5$ and $k=0.3$.

The Müller integral equations contain two types of singular integrals (see Sec. 4.6). One is a vector-valued single layer potential, and the other contains a double layer potential and another singular integral. We will state smoothing and correction results for each of these.

Proposition 6.1. With $G, G_{\delta}$, and $\partial \Omega$ as defined, and with $\mathbf{x}^{\prime} \in \partial \Omega$,

$$
\begin{aligned}
\int_{\partial \Omega}\left[\mathbf{n}\left(\mathbf{x}^{\prime}\right) \times \mathbf{f}(\mathbf{x})\right] G\left(\mathbf{x}^{\prime}-\mathbf{x}\right) d S(\mathbf{x}) & =\int_{\partial \Omega}\left[\mathbf{n}\left(\mathbf{x}^{\prime}\right) \times \mathbf{f}(\mathbf{x})\right] G_{\delta}\left(\mathbf{x}^{\prime}-\mathbf{x}\right) d S(\mathbf{x}) \\
& +\frac{\mathbf{n}\left(\mathbf{x}^{\prime}\right) \times \mathbf{f}\left(\mathbf{x}^{\prime}\right)}{\sqrt{\pi}} \delta+O\left(\delta^{3}\right) .
\end{aligned}
$$

Proposition 6.2. With $G, G_{\delta}$, and $\partial \Omega$ as defined, with $\mathbf{x}^{\prime} \in \partial \Omega$, with $H$ the mean 
curvature of the surface $\partial \Omega$, and with $D N$ the differential of the Gauss map of $\partial \Omega$,

$$
\begin{aligned}
& \int_{\partial \Omega} \mathbf{n}\left(\mathbf{x}^{\prime}\right) \times\left[\mathbf{f}(\mathbf{x}) \times \nabla G\left(\mathbf{x}^{\prime}-\mathbf{x}\right)\right] d S(\mathbf{x}) \\
= & \int_{\partial \Omega} \mathbf{n}\left(\mathbf{x}^{\prime}\right) \times\left[\mathbf{f}(\mathbf{x}) \times \nabla G_{\delta}\left(\mathbf{x}^{\prime}-\mathbf{x}\right)\right] d S(\mathbf{x}) \\
& -\frac{H\left(\mathbf{x}^{\prime}\right)+D N_{\mathbf{x}^{\prime}}}{\sqrt{\pi}} \mathbf{f}\left(\mathbf{x}^{\prime}\right) \delta+O\left(\delta^{3}\right) .
\end{aligned}
$$

Appendix B outlines the proof of these two propositions.

6.1. Discretization error. Since the integrands are periodic, we evaluate our integrals numerically with the trapezoid rule. This discretization of the integrals introduces another error. In [5], it is shown that an analogous discretization error is small as long as $\delta$ is larger than the grid spacing $h=\frac{2 \pi}{N}$ and the geometry is well represented by the grid. We therefore have reason to believe that the discretization error is small, a belief that is consistent with our results (see Sec. 8).

6.2. The corrected Müller integral equations. We present in summary the correction results of this section as they appear in the integral Equs. (4.15):

$$
\begin{aligned}
& \mathbf{j}_{i n c}\left(\mathbf{x}^{\prime}\right)=\frac{\mu_{i n t}+\mu_{\text {ext }}}{2 \mu_{\text {ext }}} \mathbf{j}_{\text {int }}\left(\mathbf{x}^{\prime}\right) \\
& -\frac{1}{\mu_{e x t}} \int_{\partial \Omega} \mathbf{n}\left(\mathbf{x}^{\prime}\right) \times\left[\mathbf{j}_{i n t}(\mathbf{x}) \times \nabla\left(\mu_{e x t} G_{\delta}^{e x t}-\mu_{i n t} G_{\delta}^{i n t}\right)\right] d S(\mathbf{x}) \\
& +\frac{\left(\mu_{e x t}-\mu_{i n t}\right)}{\mu_{e x t} \sqrt{\pi}}\left(H\left(\mathbf{x}^{\prime}\right)+D N_{\mathbf{x}^{\prime}}\right) \mathbf{j}_{i n t}\left(\mathbf{x}^{\prime}\right) \delta \\
& -\frac{i}{\mu_{e x t} \omega} \int_{\partial \Omega}\left[\mathbf{n}\left(\mathbf{x}^{\prime}\right) \times \mathbf{j}_{i n t}^{\prime}(\mathbf{x})\right]\left(k_{e x t}^{2} G_{\delta}^{e x t}-k_{i n t}^{2} G_{\delta}^{i n t}\right) d S(\mathbf{x}) \\
& -\frac{i\left(k_{e x t}^{2}-k_{i n t}^{2}\right)}{\mu_{e x t} \omega \sqrt{\pi}}\left(\mathbf{n}\left(\mathbf{x}^{\prime}\right) \times \mathbf{j}^{\prime}{ }_{i n t}\left(\mathbf{x}^{\prime}\right)\right) \delta \\
& -\frac{i}{\mu_{e x t} \omega} \int_{\partial \Omega} \mathbf{n}\left(\mathbf{x}^{\prime}\right) \times\left[\left(\mathbf{j}_{i n t}^{\prime}(\mathbf{x}) \cdot \nabla\right) \nabla\left(G^{e x t}-G^{i n t}\right)\right] d S(\mathbf{x}) \\
& +O\left(\delta^{3}\right) \text {, } \\
& \mathbf{j}_{i n c}^{\prime}\left(\mathbf{x}^{\prime}\right)=\frac{\epsilon_{i n t}+\epsilon_{e x t}}{2 \epsilon_{e x t}} \mathbf{j}_{\text {int }}^{\prime}\left(\mathbf{x}^{\prime}\right) \\
& -\frac{1}{\epsilon_{e x t}} \int_{\partial \Omega} \mathbf{n}\left(\mathbf{x}^{\prime}\right) \times\left[\mathbf{j}_{i n t}^{\prime}(\mathbf{x}) \times \nabla\left(\epsilon_{e x t} G_{\delta}^{e x t}-\epsilon_{i n t} G_{\delta}^{i n t}\right)\right] d S(\mathbf{x}) \\
& +\frac{\left(\epsilon_{\text {ext }}-\epsilon_{\text {int }}\right)}{\epsilon_{\text {ext }} \sqrt{\pi}}\left(H\left(\mathbf{x}^{\prime}\right)+D N_{\mathbf{x}^{\prime}}\right) \mathbf{j}_{\text {int }}^{\prime}\left(\mathbf{x}^{\prime}\right) \delta \\
& -\frac{i}{\epsilon_{e x t} \omega} \int_{\partial \Omega}\left[\mathbf{n}\left(\mathbf{x}^{\prime}\right) \times \mathbf{j}_{\text {int }}(\mathbf{x})\right]\left(k_{\text {ext }}^{2} G_{\delta}^{e x t}-k_{\text {int }}^{2} G_{\delta}^{i n t}\right) d S(\mathbf{x}) \\
& -\frac{i\left(k_{e x t}^{2}-k_{i n t}^{2}\right)}{\epsilon_{e x t} \omega \sqrt{\pi}}\left(\mathbf{n}\left(\mathbf{x}^{\prime}\right) \times \mathbf{j}_{i n t}\left(\mathbf{x}^{\prime}\right)\right) \delta \\
& -\frac{i}{\epsilon_{\text {ext }} \omega} \int_{\partial \Omega} \mathbf{n}\left(\mathbf{x}^{\prime}\right) \times\left[\left(\mathbf{j}_{\text {int }}(\mathbf{x}) \cdot \nabla\right) \nabla\left(G^{e x t}-G^{i n t}\right)\right] d S(\mathbf{x}) \\
& +O\left(\delta^{3}\right) \text {. }
\end{aligned}
$$


The constant $H\left(\mathbf{x}^{\prime}\right)$ and the matrix vector product $D N_{\mathbf{x}^{\prime}} \mathbf{j}$ can be evaluated as outlined in [10].

\section{Implementation}

For numerical ease, we have only implemented the method of this section on surfaces that are graphs - surfaces where $z$ is a function of $x$ and $y$. We have also only implemented the method with normally incident light.

There are some details in the numerical implementation of our solution to the scattering problem that need to be mentioned.

7.1. Special functions. Computing the Green's functions requires the evaluation of many exponential integrals. These can be evaluated according to the iterative relationships described in Appendix A. Thus, computation of the exponential integral of any order will require evaluation of the complex complementary error function erfc. We have implemented the algorithm of [25] for this purpose.

7.2. Evaluating the smoothed Green's functions. The smoothed Green's function introduced in Sec. 6 must be written in in terms of exponential integral functions for implementation:

$$
\begin{aligned}
G 1_{\delta}\left(\mathbf{x}^{\prime}-\mathbf{x}\right) & =\frac{1}{8 \pi^{3 / 2}} \sum_{\mu, \nu} e^{-2 \pi i(\alpha \mu+\beta \nu)} \\
& \times \sum_{\ell=0}^{\infty} \frac{k^{2 \ell}}{\ell !}\left(E^{2 \ell-1} \operatorname{ExpInt}_{\ell+1 / 2}\left(\frac{R_{\mu \nu}^{2}}{4 E^{2}}\right)-\delta^{2 \ell-1} \operatorname{ExpInt}_{\ell+1 / 2}\left(\frac{R_{\mu \nu}^{2}}{4 \delta^{2}}\right)\right) .
\end{aligned}
$$

We can thus compute $G_{\delta}$ and can similarly compute all of the needed derivatives of the Green's function.

We must be able to evaluate $G_{\delta}$ at the point $\mathbf{x}^{\prime}-\mathbf{x}=\mathbf{0}$. This is, of course, straightforward for the smooth $G 2$ part of the Green's function. The $G 1_{\delta}$, however,

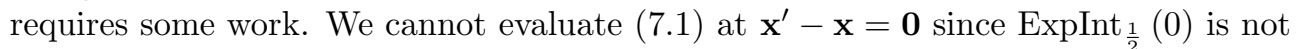
defined. Only the $\mu=\nu=0$ term is problematic, and we can find this term exactly using the representation of (6.1) and integrating:

$$
\frac{1}{8 \pi^{3 / 2}} \int_{\delta^{2}}^{E^{2}} \frac{e^{k^{2} t}}{t^{3 / 2}} d t=\frac{1}{4 \pi^{3 / 2}}\left(\frac{e^{k^{2} \delta^{2}}}{\delta}-\frac{e^{k^{2} E^{2}}}{E}\right)+\frac{k i}{4 \pi}[\operatorname{erfc}(i E k)-\operatorname{erfc}(i \delta k)] .
$$

The remaining terms (those for $\mu$ and $\nu$ other than $\mu=\nu=0$ ) can be computed as in (7.1). For most of the needed derivatives of $G_{\delta}$, the $\mu=\nu=0$ term in the summation will be 0 when evaluated at $\mathbf{x}^{\prime}-\mathbf{x}=\mathbf{0}$. However, in the second derivatives $G_{\delta_{x x}}$, $G_{\delta_{y y}}$, and $G_{\delta_{z z}}$, there is a nonzero contribution. We will illustrate with an integral that appears in $G_{\delta_{x x}}(\mathbf{0})$, which we can again integrate exactly:

$$
\begin{aligned}
-\frac{1}{16 \pi^{3 / 2}} \int_{\delta^{2}}^{E^{2}} \frac{e^{k^{2} t}}{t^{5 / 2}} d t= & -\frac{1}{24 \pi^{3 / 2}}\left(\frac{\left(1+2 k^{2} \delta^{2}\right) e^{k^{2} \delta^{2}}}{\delta^{3}}-\frac{\left(1+2 k^{2} E^{2}\right) e^{k^{2} E^{2}}}{E^{3}}\right) \\
& +-\frac{k^{3} i}{12 \pi}[\operatorname{erfc}(i E k)-\operatorname{erfc}(i \delta k)] .
\end{aligned}
$$

This integral also appears in $G_{\delta_{y y}}(\mathbf{0})$ and $G_{\delta_{z z}}(\mathbf{0})$. The other terms in these second derivatives can be computed through exponential integral functions as in (7.1). 
7.3. Size. The drawback to using integral equations methods in scattering is that they lead to large, dense linear systems. Although our system is well-posed, it can become too large for practical computing purposes. With our scatterer described by an $N \times N$ grid in $x$ and $y$, we must solve a complex-valued $12 N^{2} \times 12 N^{2}$ linear system.

7.4. Acceleration techniques. There are various ways in which we can improve the speed of the numerical approximations. For surfaces defined by $z=$ $f(x, y)$, the $G 2$ sums over $m$ and $n$ can be computed with FTTs and stored. This leads to considerable savings, and it allows us to use a smaller Ewald splitting parameter $E$ to also accelerate the $G 1$ sums (see Sec. 5 ). We precompute and store many quantities from the Green's functions that are used repeatedly, and this also is a valuable time saver. There is, of course, a natural symmetry in the Green's functions that can be exploited since $G\left(\mathbf{x}-\mathbf{x}^{\prime}\right)=G\left(\mathbf{x}^{\prime}-\mathbf{x}\right)$. Finally, we have employed cubic-spline interpolation to approximate values of the exponential integral functions $\operatorname{ExpInt}_{n}(x)$. This interpolation requires little storage, and it leads to a less expensive evaluation of $\operatorname{ExpInt}_{n}(x)$. We have found it to be worthwhile.

7.5. GMRES. We have implemented the generalized minimal residual method (GMRES) since it does not require storage of the full $144 N^{4}$ entry matrix. We have found that the number of iterations required is influenced by both the geometry of the scatterer and the presence or absence of resonances in the scatterer (see Sec. 8 for details). Still, in worst cases, seldom are more than 100 iterations needed for convergence within our tolerance of $10^{-4}$ using the convergence criteria of [19].

We have implemented a restarted GMRES, but the restarts tended to slow convergence dramatically. Standard preconditioning matrices are known to be unsuccessful in electromagnetic scattering problems in general, and preconditioning is considered an open problem in this field [22]. Most preconditioning strategies that have been implemented are to be used in conjunction with fast summation methods [7].

Following [2], we know that the linear system is well-conditioned except possibly near frequencies that are eigenvalues. From the 2D work of [26] and [27], we have reason to believe that the eigenvalues of the system have small imaginary parts. The resonances that we observe and discuss in Chapter 8 are believed to occur for real frequencies near these complex eigenvalues. We would therefore expect the linear system to have higher condition numbers near the resonances. This is precisely what has been observed (see Fig. 8.3), as the system requires more GMRES iterations at frequencies near resonances.

\section{Results}

We have implemented the numerical scattering problem as described with various geometries and under various conditions. Here we outline some of the results. In all cases, we have observed convergence that appears to be third order in the grid size $h=\frac{2 \pi}{N}$.

8.1. Integrals on curved surfaces. A large part of this paper is the method of smoothing and correcting singular integrals developed in Sec. 6. In order to test our method independent of the integral equation and the scattering problem, we have evaluated all of Müller's integrals on the surface defined by

$$
F_{1}(x, y)=C \cos ^{2}\left(\frac{x}{2}\right) \sin ^{2}\left(\frac{y}{2}\right) \quad \text { and } \quad F_{2}(x, y)=L-C \cos ^{2}\left(\frac{x}{2}\right) \sin ^{2}\left(\frac{y}{2}\right) .
$$




\begin{tabular}{|c|c|c|}
\hline point $\left(x^{\prime}, y^{\prime}\right)$ & slp ratio $\mathrm{R}$ & $d l p$ ratio $\mathrm{R}$ \\
\hline \hline$(0,0)$ & 8.05 & 7.63 \\
\hline$(\pi, \pi)$ & 7.99 & 7.89 \\
\hline$\left(\frac{\pi}{2}, \frac{\pi}{2}\right)$ & 7.94 & 7.60 \\
\hline$\left(\frac{\pi}{8}, \frac{\pi}{4}\right)$ & 8.22 & 7.40 \\
\hline$\left(\frac{5 \pi}{8}, \frac{7 \pi}{4}\right)$ & 7.97 & 7.82 \\
\hline$\left(\frac{\pi}{2}, \frac{3 \pi}{4}\right)$ & 7.79 & 7.90 \\
\hline
\end{tabular}

Table 8.1. Table of Results for Single and Double Layer Potentials on a Smooth Dimpled Surface.

The results presented here are for $L=2$ and $C=\frac{1}{5}$. We call this surface the smoothed, dimpled scatterer.

We perform the smoothing and correction method for both a single and a double layer potential with the density function $\phi(\mathbf{x})=\cos (x) \cos (y)$. We have evaluated each at various points $\mathbf{x}^{\prime}$ over the surface (8.1). The smoothed Green's functions were integrated, and the correction terms were added to the sums. The integrals were evaluated with the Trapezoid Rule with $N=64, N=128$, and $N=256$. A sample of typical results are reported here in Table 8.1. The values reported are the ratios

$$
R=\frac{I(4 h)-I(2 h)}{I(2 h)-I(h)} .
$$

A ratio close to 8 would be consistent with third-order convergence. The results in the table are a small sample of typical results for wave numbers $k$ in the range $k=0.8$ to $k=1.8$ and smoothing parameters $\delta$ in the range $\delta=h$ to $\delta=3 h$.

We have found similar convergence results for all of the integrals needed in Müller's integral equations. Without correction terms, the ratios for the singular integrals are very close to 2 , indicating first order convergence. We have found similar convergence results for each of the other scatterers described below.

8.2. A flat slab scattering problem. We can use a flat slab geometry with normally incident fields as a test case, since an exact analytical solution is known. For this problem, with $\mathbf{E}_{i n c}=\left(e^{i k z}, 0,0\right)$, the transmission coefficient is

$$
T(\omega)=4 \theta \sqrt{\frac{1}{(1+\theta)^{4}+(1-\theta)^{4}-2\left(1-\theta^{2}\right)^{2} \cos \left(\frac{2 \omega L}{\theta}\right)}}
$$

where $\theta=\sqrt{1 / \epsilon_{\text {int }}}$. See [21] for a derivation of this result.

8.3. Results in the flat slab test case. We have implemented the numerical scattering experiment for the flat slab scatterer with various physical and numerical parameters. In all cases, the agreement is excellent. We present the results for $\epsilon_{\text {int }}=5$ and $N=32$ in Fig. 8.1, where we see extremely good agreement. Fig. 8.1 also includes results for the scattering experiment when the correction terms in the integral equations are omitted. The difference is striking and illustrates the effect of the corrections. We have done approximations with $N$ as low as 16, and even such a coarse grid gives very good results for the test case.

Fig. 8.2 gives results for $\epsilon_{i n t}=15$. This is a more difficult case, since the frequency is higher and convergence of the Green's functions is therefore slower. The results are still very good. We have also approximated transmission coefficients for lossy 

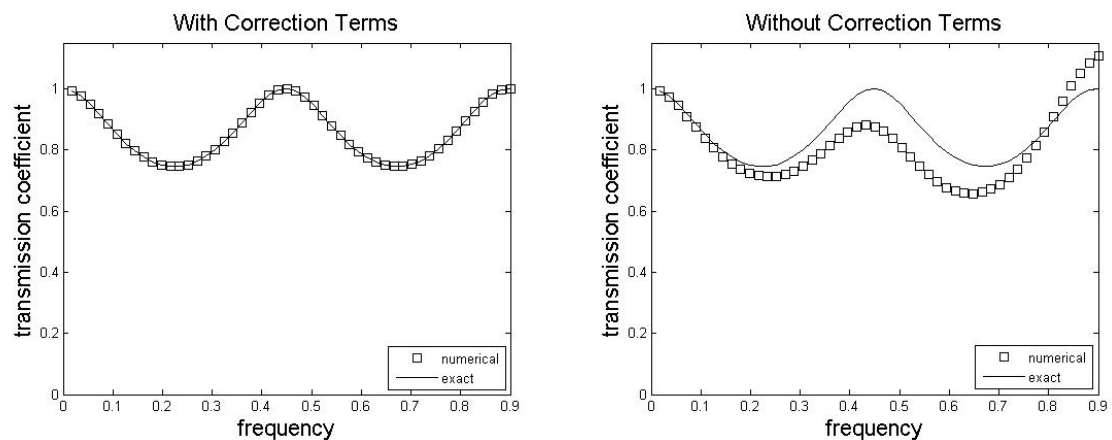

FiG. 8.1. The flat slab test case. On the left are the numerical approximations with corrections. On the right, the corrections have been omitted. $N=32 . \epsilon=5 . \delta=h$.

scatterers - scatterers where $\epsilon_{\text {int }}$ has an imaginary part. The results for $\epsilon_{\text {int }}=15+0.5 i$ are also in Fig. 8.2.
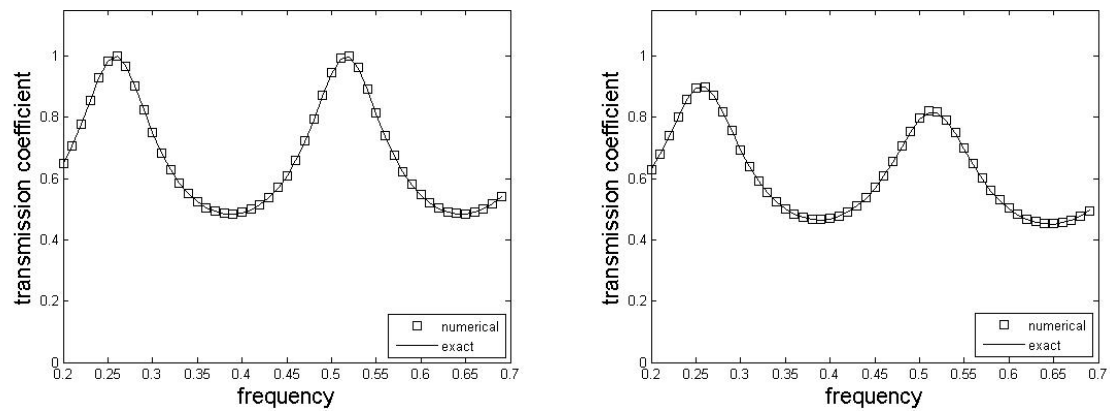

FiG. 8.2. Two more results for the flat slab test case. On the left are results for a higher dielectric contrast $\epsilon=15$. On the right are results for a lossy scatterer $\epsilon=15+0.5 i$. For each, $N=32 . \delta=h$.

8.4. Smooth dimpled scatterer. Results for the smooth, dimpled scatterer (8.1) with $L=\pi$ are in Fig. 8.3. We note interesting behavior at some frequencies, most notably a sharp dip in transmission around $\omega=0.57$. In general for our results, the interesting features in transmission occur at frequencies for which a $\lambda_{m n}$ mode of the Green's function is close to zero. This behavior is not unexpected and is related to the Wood's anomaly (see Sec. 3.2). Fig. 8.3 shows the number of GMRES iterations required to solve the linear system at each frequency. It is clear that iterations increase with frequency, and it appears that more iterations are required for frequencies near resonances (see Sec. 7.5). We observe third order convergence in these results.

8.5. Corrugated roof surface. The most interesting surface that we have experimented with thus far is the corrugated roof surface:

$$
F_{1}(x, y)=C \sin ^{2}\left(\frac{x}{2}\right) \quad \text { and } \quad F_{2}(x, y)=L-C \sin ^{2}\left(\frac{x}{2}\right) .
$$

This is basically a 2D dimple, as the surface has no $y$ dependence. The results for $C=\frac{1}{5}$ and $L=\pi$ are seen in Fig. 8.4, where we see the interesting behavior of the 

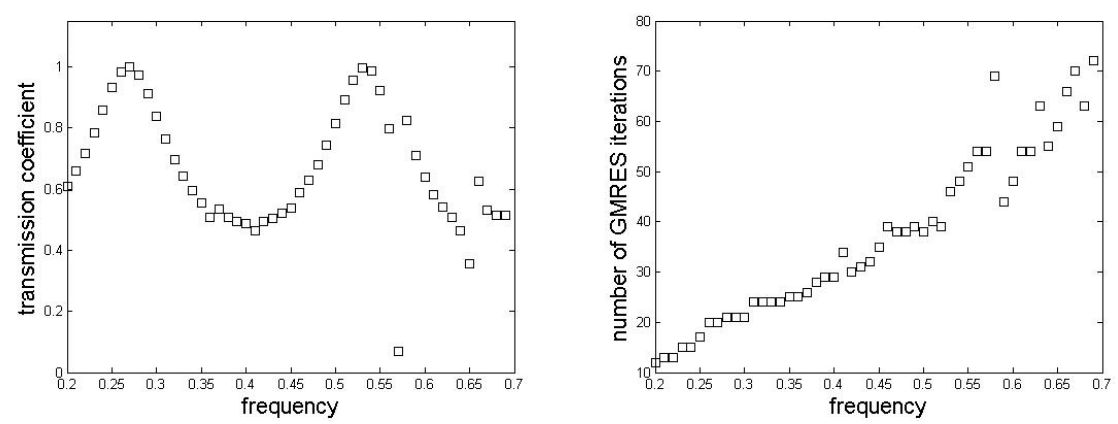

FIG. 8.3. On the left are transmission coefficients for the smooth dimpled scatterer. On the right are the number of GMRES iterations required for each frequency. $\epsilon=15 . N=32 . \delta=h$.

corrugated roof scatterer. There are two clear resonances in our evaluated frequency range. One is centered near $\omega=0.37$ and the other near $\omega=0.58$. Once again these resonances are examples of Wood's anomaly (3.2). Wood's anomalies have been observed in scattering experiments where $\lambda_{m n}^{e x t}$ is near $0[34,15]$. Here, we observe it for frequencies where $\lambda_{m n}^{i n t}$ is near 0 . Such an internal Wood's anomaly has previously been unknown. The resonance centered at $\omega=0.37$ is particularly interesting, since there is a transmission spike up to full transmission.
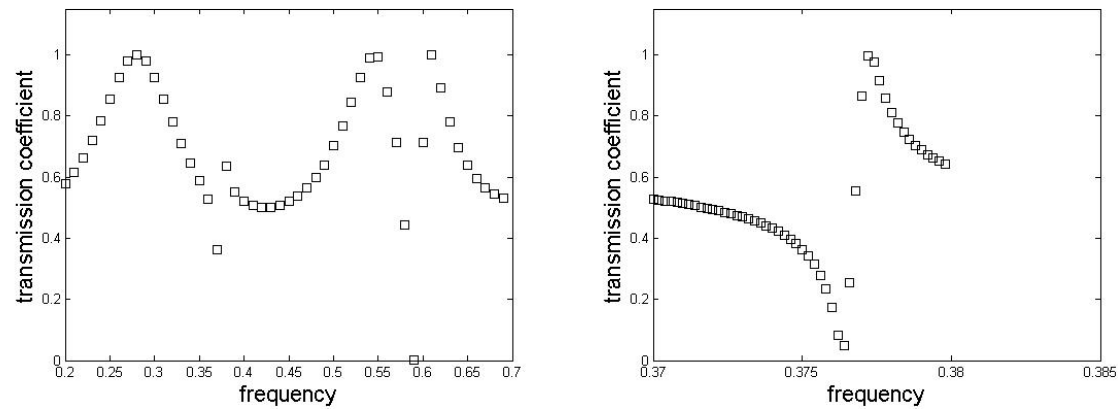

FIG. 8.4. Transmission coefficients for the corrugated roof scatterer. On the right we have zoomed in for a closer look at the $\omega=0.37$ resonance. $\epsilon=15 . N=32 . \delta=h$.

8.6. Discussion of results. The results are promising. We see very good agreement with an exact test case, and we see third order convergence for most frequencies on curved surfaces. We have seen also that the method is capable of resolving such interesting physical phenomena as transmission resonances. Comparing with results from a first order method [3], we can see the advantage of being able to approximate with $O\left(h^{3}\right)$ accuracy.

Appendix A. Exponential integral functions.

The exponential integral of order $n$ is defined as

$$
\operatorname{ExpInt}_{n}(x)=\int_{1}^{\infty} \frac{e^{-x t}}{t^{n}} d t
$$


for $\operatorname{Re}(x)>0$. The exponential integral functions that appear in our Green's functions are all of half integer orders of at least $-\frac{1}{2}$. The following facts allow us to compute all needed exponential integral functions:

$$
\begin{aligned}
& \operatorname{ExpInt}_{\frac{1}{2}}(x)=\sqrt{\frac{\pi}{x}} \operatorname{erfc}(\sqrt{x}) \\
& \text { ExpInt }_{-\frac{1}{2}}(x)=\frac{1}{x}\left[\operatorname{ExpInt}_{\frac{1}{2}}(x)+e^{-x}\right] \\
& \operatorname{ExpInt}_{n+1}(x)=\frac{1}{n}\left[e^{-x}-x \operatorname{ExpInt}_{n}(x)\right] \\
& \frac{d}{d x}\left[\operatorname{ExpInt}_{n}(x)\right]=-\operatorname{ExpInt}_{n-1}(x)
\end{aligned}
$$

We see that all of the exponential integral functions we need are related to the complementary error function erfc and to square root functions.

For order $n \leq 1$, exponential integral functions are singular as $x \rightarrow 0$. For $n>1$, ExpInt $_{n}(x)$ is finite at $x=0$. By looking at (A.2), we can see the exact nature of the singularities that we will encounter.

$$
\begin{array}{rlrl}
\operatorname{ExpInt}_{\frac{1}{2}}(x) & \rightarrow \sqrt{\frac{\pi}{x}} \quad \text { as } & x \rightarrow 0 \\
\operatorname{ExpInt}_{-\frac{1}{2}}(x) & \rightarrow \frac{\sqrt{\pi}}{x^{3 / 2}} \quad \text { as } & & x \rightarrow 0 .
\end{array}
$$

The exponential integral function of any order decays quickly as $\operatorname{Re}(() x)$ increases. This decay ensures quick convergence of the Green's function terms after Ewald splitting (see Sec. 5). For $x>1$ and for any half integer $n \geq-\frac{1}{2}$, we have

$$
\operatorname{ExpInt}_{n}(x) \leq \frac{2 e^{-x}}{x}
$$

Appendix B. Derivation of correction terms. We will derive the correction term for a smoothed double layer potential. The other corrections of Sec. 6 can be found in a similar manner. We are interested in investigating the error $\epsilon$ introduced by replacing the Green's function $G$ with the regularized Green's function $G_{\delta}$ in a double layer potential when the reference variable $\mathbf{x}^{\prime}$ is on the surface. We are able to identify and quantify the largest part of this error and use it as a correction to the smoothed integral. These derivations first appeared in [21].

The derivation of the correction term involves some ideas from classical differential geometry. In particular, certain changes of variables are used to simplify various expressions. The corrections can be derived without such simplifications, and such a derivation is outlined in [21].

Proposition B.1 (The Double Layer Potential). With $G, G_{\delta}$, and $\partial \Omega$ as defined, with $\mathbf{x}^{\prime} \in \partial \Omega$, and with $H$ the mean curvature of the surface $\partial \Omega$,

$$
\begin{aligned}
\int_{\partial \Omega}\left[\mathbf{n}(\mathbf{x}) \cdot \nabla G\left(\mathbf{x}^{\prime}-\mathbf{x}\right)\right] f(\mathbf{x}) d S(\mathbf{x})= & \int_{\partial \Omega}\left[\mathbf{n}(\mathbf{x}) \cdot \nabla G_{\delta}\left(\mathbf{x}^{\prime}-\mathbf{x}\right)\right] f(\mathbf{x}) d S(\mathbf{x}) \\
& +\frac{f\left(\mathbf{x}^{\prime}\right) H\left(\mathbf{x}^{\prime}\right)}{\sqrt{\pi}} \delta+O\left(\delta^{3}\right) .
\end{aligned}
$$


Proof. The error is the difference between the two integrals

$$
\epsilon=\int_{\partial \Omega} \mathbf{n}(\mathbf{x}) \cdot\left[\nabla\left(G\left(\mathbf{x}^{\prime}-\mathbf{x}\right)-G_{\delta}\left(\mathbf{x}^{\prime}-\mathbf{x}\right)\right)\right] f(\mathbf{x}) d S(\mathbf{x}) .
$$

Without loss of generality, we take $\mathbf{x}^{\prime}=\mathbf{0}$. The error becomes:

$$
\epsilon=\frac{1}{8 \pi^{3 / 2}} \int_{\partial \Omega}\left(\sum_{\mu, \nu} \int_{0}^{\delta^{2}} \mathbf{n}(\mathbf{x}) \cdot \nabla\left(\frac{e^{k^{2} t-\frac{R_{\mu \nu}^{2}}{4 t}}}{t^{3 / 2}}\right) d t\right) f(\mathbf{x}) d S(\mathbf{x}) .
$$

The error decays very quickly as $R_{\mu \nu}$ increases. For this reason, we need only include the $\mu=\nu=0$ term in the double sum. We are left with

$$
\epsilon=-\frac{1}{16 \pi^{3 / 2}} \int_{0}^{\delta^{2}} \frac{e^{k^{2} t}}{t^{5 / 2}}\left(\int_{\partial \Omega}[\mathbf{n}(\mathbf{x}) \cdot \mathbf{x}] e^{-\frac{r^{2}}{4 t}} f(\mathbf{x}) d S(\mathbf{x})\right) d t .
$$

Again, the error is highly concentrated around the singular point $r=|\mathbf{x}|=0$. The contribution to the error away from the singularity is negligible. We can perform the surface integral on only a small set of $\partial \Omega$ including $r=0$ and still find the leading order term of the error. We let $D$ be an open set in $\mathbb{R}^{2}$ with coordinates $\alpha=\left(\alpha_{1}, \alpha_{2}\right)$ such that $\alpha$ can be smoothly (at least $C^{3}$ ) mapped from $D$ onto an open set in $\partial \Omega$. Then $\mathbf{x} \in \partial \Omega$ becomes $\mathbf{x}(\alpha)$. We choose this coordinate patch so that $\mathbf{x}(0)=\mathbf{0}$. The error can now be written as an integral in $\alpha$ over $D$, which we extend (without adding meaningful error) to an integral over all of $\mathbb{R}^{2}$ :

$$
\epsilon=-\frac{1}{16 \pi^{3 / 2}} \int_{0}^{\delta^{2}} \frac{e^{k^{2} t}}{t^{5 / 2}}\left(\int_{\mathbb{R}^{2}}[\mathbf{n}(\alpha) \cdot \mathbf{x}(\alpha)] e^{-\frac{r(\alpha)^{2}}{4 t}} f(\alpha)\left|\frac{\partial \mathbf{x}}{\partial \alpha_{1}} \times \frac{\partial \mathbf{x}}{\partial \alpha_{2}}\right| d \alpha\right) d t
$$

As outlined in [1] and [5], we can choose (rotating and translating as necessary) coordinates $\alpha$ so that the Christoffel symbols are zero at $\mathbf{x}=\mathbf{0}$. We then make a linear change of variables so that the tangential vectors $\mathbf{T}_{\mathbf{j}}=\frac{\partial \mathbf{x}}{\partial \alpha_{j}}$ point in the directions of principal curvature of the surface and the metric tensor is the identity at $\mathbf{x}=\mathbf{0}$. We call these vectors the principal directions. We then have an orthonormal coordinate system $\left(\mathbf{T}_{\mathbf{1}}(\mathbf{0}), \mathbf{T}_{\mathbf{2}}(\mathbf{0}), \mathbf{n}_{\mathbf{0}}\right)$. The error integral becomes:

$$
\epsilon=-\frac{1}{16 \pi^{3 / 2}} \int_{0}^{\delta^{2}} \frac{e^{k^{2} t}}{t^{5 / 2}}\left(\int_{\mathbb{R}^{2}}[\mathbf{n}(\alpha) \cdot \mathbf{x}(\alpha)] e^{-\frac{r(\alpha)^{2}}{4 t}} f(\alpha)\left|\mathbf{T}_{\mathbf{1}} \times \mathbf{T}_{\mathbf{2}}\right| d \alpha\right) d t .
$$

Following [1] and [5], we expand $\mathbf{x}(\alpha)$ and $\mathbf{n}(\alpha)$ in Taylor series around $\alpha=0$. What makes the new coordinate system nice is that there is no $\alpha_{1} \alpha_{2}$ term among the second order terms:

$$
\begin{aligned}
& \mathbf{x}(\alpha)=\mathbf{T}_{\mathbf{1}}(0) \alpha_{1}+\mathbf{T}_{\mathbf{2}}(0) \alpha_{2}+\frac{1}{2} \kappa_{1} \mathbf{n}_{\mathbf{0}} \alpha_{1}^{2}+\frac{1}{2} \kappa_{2} \mathbf{n}_{\mathbf{0}} \alpha_{2}^{2}+O\left(|\alpha|^{3}\right) \\
& \mathbf{n}(\alpha)=\mathbf{n}_{\mathbf{0}}-\kappa_{1} \mathbf{T}_{\mathbf{1}} \alpha_{1}-\kappa_{2} \mathbf{T}_{\mathbf{2}} \alpha_{2}+O\left(|\alpha|^{2}\right)
\end{aligned}
$$

where $\kappa_{1}$ and $\kappa_{2}$ are the principal curvatures at $\mathbf{x}^{\prime}=\mathbf{0}$. Then

$$
r^{2}=\mathbf{x}(\alpha) \cdot \mathbf{x}(\alpha)=|\alpha|^{2}+O\left(|\alpha|^{4}\right)
$$

and

$$
\mathbf{x}(\alpha) \cdot \mathbf{n}(\alpha)=-\frac{1}{2} \kappa_{1} \alpha_{1}^{2}-\frac{1}{2} \kappa_{2} \alpha_{2}^{2}+O\left(|\alpha|^{3}\right)
$$


Here (and throughout this section), we make use of the inequality $\alpha_{j}^{2} \leq|\alpha|^{2}$.

We now perform a change of variables $\alpha \rightarrow \xi$ such that $|\xi|^{2}=r^{2}$ and $\xi_{i} /|\xi|=$ $\alpha_{i} /|\alpha|$. This change is essential to our analysis. Without it, there are quickly changing high order terms in the exponential as $t \rightarrow 0$. The substitution eliminates these terms from the exponential while allowing $\xi$ to have the same direction as $\alpha$. From (B.1)

$$
|\xi|^{2}=|\alpha|^{2}+O\left(|\alpha|^{4}\right)
$$

and thus

$$
|\xi|=|\alpha| \sqrt{1+O\left(|\alpha|^{2}\right)}=|\alpha|+O\left(|\alpha|^{3}\right) .
$$

Since $O\left(|\alpha|^{3}\right)=O\left(|\xi|^{3}\right)$ we have

$$
|\alpha|=|\xi|+O\left(|\xi|^{3}\right)
$$

and then

$$
\alpha_{i}=\xi_{i}+O\left(|\xi|^{3}\right)
$$

The error is now

$$
\epsilon=-\frac{1}{16 \pi^{3 / 2}} \int_{0}^{\delta^{2}} \frac{e^{k^{2} t}}{t^{5 / 2}}\left(\int_{\mathbb{R}^{2}}[\mathbf{n}(\xi) \cdot \mathbf{x}(\xi)] e^{-\frac{|\xi|^{2}}{4 t}} f(\xi) \operatorname{det}(\partial \alpha / \partial \xi)\left|\mathbf{T}_{\mathbf{1}} \times \mathbf{T}_{\mathbf{2}}\right| d \xi\right) d t
$$

The dot product in the integrand above becomes

$$
\mathbf{x} \cdot \mathbf{n}(\mathbf{x})=-\frac{1}{2} \kappa_{1} \xi_{1}^{2}-\frac{1}{2} \kappa_{2} \xi_{2}^{2}+O\left(|\xi|^{3}\right) .
$$

We expand $f(\xi)$ :

$$
f(\xi)=f(0)+f_{j}(0) \xi_{j}+f_{i j}(0) \xi_{i} \xi_{j}+O\left(|\xi|^{3}\right)
$$

with the terms being summed over $i$ and $j$. Using (B.3), we see that:

$$
\operatorname{det}(\partial \alpha / \partial \xi)=1+O\left(|\xi|^{2}\right) \text {. }
$$

Since $\mathbf{T}_{\mathbf{j}}(\alpha)=\frac{\partial \mathbf{x}}{\partial \alpha_{j}}$, we have

$$
\left|\mathbf{T}_{\mathbf{1}} \times \mathbf{T}_{\mathbf{2}}\right|=1+O\left(|\xi|^{2}\right) .
$$

The integrand of the $d \xi$ integral becomes:

$$
e^{-\frac{|\xi|^{2}}{4 t}}\left(-\frac{1}{2} \kappa_{1} \xi_{1}^{2} f(0)-\frac{1}{2} \kappa_{2} \xi_{2}^{2} f(0)+R(\xi)\right)
$$

where, by the Taylor series remainder theorem, the remainder function $R(\xi)$ has the form

$$
R(\xi)=C_{1} \xi_{1}^{3}+C_{2} \xi_{2}^{3}+C_{3} \xi_{1}^{2} \xi_{2}+C_{4} \xi_{1} \xi_{2}^{2}
$$

for constants $C_{1}, C_{2}, C_{3}$, and $C_{4}$. Each of these terms, however, is odd and will integrate to zero. The remainder function must really be $O\left(|\xi|^{4}\right)$

$$
R(\xi)=C_{1} \xi_{1}^{4}+C_{2} \xi_{2}^{4}+C_{3} \xi_{1}^{3} \xi_{2}+C_{4} \xi_{1} \xi_{2}^{3}+C_{5} \xi_{1}^{2} \xi_{2}^{2} .
$$


The $C_{3} \xi_{1}^{3} \xi_{2}$ and $C_{4} \xi_{1} \xi_{2}^{3}$ terms are odd in $\xi_{i}$ and will also integrate to zero, and we drop them. We make the change of variables $\xi=\delta \zeta$. We write the remainder function as $R(\xi)=\delta^{4}\left(C_{1} \zeta_{1}^{4}+C_{2} \zeta_{2}^{4}+C_{5} \zeta_{1}^{2} \zeta_{2}^{2}\right)=\delta^{4} \tilde{R}(\zeta)$. The error is now

$$
\begin{aligned}
\epsilon & =\frac{\delta^{4}}{16 \pi^{3 / 2}} \int_{0}^{\delta^{2}} \frac{e^{k^{2} t}}{t^{5 / 2}}\left(\int_{\mathbb{R}^{2}} e^{-\frac{\delta^{2}|\zeta|^{2}}{4 t}}\left(\frac{1}{2} \kappa_{1} \zeta_{1}^{2} f(0)+\frac{1}{2} \kappa_{2} \zeta_{2}^{2} f(0)+\delta^{2} \tilde{R}(\zeta)\right) d \zeta\right) d t \\
& =\frac{f(0) \delta^{4}}{32 \pi^{3 / 2}} \int_{0}^{\delta^{2}} \frac{e^{k^{2} t}}{t^{5 / 2}}\left(\int_{\mathbb{R}^{2}}\left(\kappa_{1} \zeta_{1}^{2}+\kappa_{2} \zeta_{2}^{2}\right) e^{-\frac{\delta^{2}|\zeta|^{2}}{4 t}} d \zeta\right) d t+I_{\tilde{R}} .
\end{aligned}
$$

We will change to polar coordinates. Let $|\zeta|=s, \zeta_{1}=s \cos \theta$, and $\zeta_{2}=s \sin \theta$ :

$$
\begin{aligned}
\epsilon & =\frac{f(0)\left(\kappa_{1}+\kappa_{2}\right) \delta^{4}}{32 \pi^{1 / 2}} \int_{0}^{\delta^{2}} \frac{e^{k^{2} t}}{t^{5 / 2}}\left(\int_{0}^{\infty} s^{3} e^{-\frac{\delta^{2} s^{2}}{4 t}} d s\right) d t+I_{\tilde{R}} \\
& =\frac{f(0)\left(\kappa_{1}+\kappa_{2}\right)}{4 \pi^{1 / 2}} \int_{0}^{\delta^{2}} \frac{e^{k^{2} t}}{t^{1 / 2}} d t+I_{\tilde{R}} \\
& =\frac{f(0) H(0)}{\pi^{1 / 2}} \delta+O\left(\delta^{3}\right)+I_{\tilde{R}}
\end{aligned}
$$

where $H=\frac{\kappa_{1}+\kappa_{2}}{2}$ is the mean curvature of the surface.

We examine the $\tilde{R}$ integral, $I_{\tilde{R}}$, in the same way and obtain:

$$
I_{\tilde{R}}=-\frac{\delta^{6}}{16 \pi^{3 / 2}} \int_{0}^{\delta^{2}} \frac{e^{k^{2} t}}{t^{5 / 2}}\left(\int_{\mathbb{R}^{2}}\left(C_{1} \zeta_{1}^{4}+C_{2} \zeta_{2}^{4}+C_{5} \zeta_{1}^{2} \zeta_{2}^{2}\right) e^{-\frac{\delta^{2}|\zeta|^{2}}{4 t}} d \zeta\right) d t=O\left(\delta^{3}\right)
$$

We now have the result

$$
\epsilon=\frac{f(0) H(0)}{\pi^{1 / 2}} \delta+O\left(\delta^{3}\right)
$$

\section{REFERENCES}

[1] Rutherford Aris, Vectors, tensors and the basic equations of fluid mechanics, 2nd ed., Dover Publications, 1990.

[2] Kendall E. Atkinson, The numerical solution of integral equations of the second kind, Cambridge, 1997.

[3] Andrew Barnes, Electromagnetic scattering from three dimensional periodic structures, Thesis, Duke Univeristy, 2003.

[4] J. Thomas Beale, A convergent boundary integral method for three-dimensional water waves, Math. Comput., 70(235), 977-1029, 2001.

[5] _ A grid-based boundary integral method for elliptic problems in three dimensions, SIAM J. Numer. Anal., 42(2), 599-620, (electronic) 2004.

[6] J. Thomas Beale and Ming-Chih Lai, A method for computing nearly singular integrals, SIAM J. Numer. Anal., 38(6), 1902-1925, (electronic) 2001.

[7] Angelika Bunse-Gerstner and Ignacio Gutiérrez-Cañas, A preconditioned GMRES for complex dense linear systems from electromagnetic wave scattering problems, Linear Algebra Appl., 416(1), 135-147, 2006.

[8] F. Capolino, D.R. Wilton and W.A. Johnson, Efficient computation of the 3d Green's function for the Helmholtz operator for a linear array of point sources using the Ewald method, J. Comput. Phys., 223(1), 2007.

[9] Filippo Capolino, Donald R. Wilton and William A. Johnson, Efficient computation of the 2-D Green's function for 1-D periodic structures using the Ewald method, IEEE Trans. Antennas and Propagation, 53(9), 2977-2984, 2005. 
[10] Manfredo P. do Carmo, Differential geometry of curves and surfaces, Prentice-Hall, 503, 1976.

[11] Kokou Dossou, Michael A. Byrne and Lindsay C. Botten, Finite element computation of grating scattering matrices and application to photonic crystal band calculations, J. Comput. Phys., 219(1), 2006.

[12] T.W. Ebbessen, H.J. Lezec, H.F. Ghaemi, T. Thio and P.A. Wolff, Extraordinary optical transmission through sub-wavelength hole arrays, Nature, 391(12), 667-669, 1998.

[13] P.P. Ewald, Die berechnung optischer und electrostatischer gitterpotentiale, Ann. Phys., 64, 253-287, 1921.

[14] S. Fan and J.D. Joannopoulos, Analysis of guided resonance in photonic crystal slabs, Phys. Rev. B, 65, 2002.

[15] Lan Gao and Junichi Nakayama, Scattering of a TM plane wave from periodic random surfaces, Waves Random Media, 9, 53-67, 1999.

[16] David Griffiths, Introduction to Electrodynamics, Prentice Hall, 1999.

[17] Mansoor A. Haider, Stephen P. Shipman and Stephanos Venakides, Boundary-integral calculations of two-dimensional electromagnetic scattering in infinite photonic crystal slabs: channel defects and resonances, SIAM J. Appl. Math., 62(6), 2129-2148, (electronic) 2002.

[18] Steven G Johnson, Shanhui Fan, Pierre R. Villeneuve and J.D. Joannopoulos, Guided modes in photonic crystal slabs, Phys. Rev. B, 60(8), 5751-5758, 2002.

[19] C.T. Kelley, Iterative Methods for Linear and Nonlinear Equations, SIAM, 1995.

[20] C. Müller, Foundations of the Mathematical Theory of Electromagnetic Waves, Springer, Berlin, 1969.

[21] Michael Nicholas, A third order numerical method for $3 d$ doubly periodic electromagnetic scattering problems, Thesis, Duke Univeristy, 2007.

[22] David Nicholls, Nilima Nigam and Fernando Reitich, Advances in computational scattering, Summary of Conference "Advances in Computational Scattering" at Banff International Research Station, 18-23, 2006.

[23] Vassilis G. Papanicolaou, Ewald's method revisited: rapidly convergent series representations of certain Green's functions, J. Comput. Anal. Appl., 1(1), 105-114, 1999.

[24] Giuseppe Pelosi, Alessandro Cocchi and Stafano Selleri, Electromagnetic scattering from infinite periodic structures with a localized impurity, IEEE Transactions on Antennas and Propagation, 49(5), 697-702, 2001.

[25] G.P.M. Poppe and C.M.J. Wijers, Algorithm 680: evaluation of the complex error function, ACM Trans. Math. Software, 16(1), 47, 1990.

[26] S. Shipman and S. Venakides, Resonant transmission near nonrobust periodic slab modes, Phys. Rev. E, 71, 026611, 1-10, 2005.

[27] Stephen P. Shipman and Stephanos Venakides, Resonance and bound states in photonic crystal slabs, SIAM J. Appl. Math., 64(1), 322-342, (electronic) 2003.

[28] Stephen P. Shipman and Stephanos Venakides, Resonant transmission near nonrobust periodic slab modes, Physical Review E (Statistical, Nonlinear, and Soft Matter Physics), 71(2), 026611, 2005.

[29] V.G. Solov'ev, C.M. Torres and S.G. Romanov, Reflection, transmission, and scattering of light by photonic crystals based on opal films, Russian Physics Journal, 47(3), 286-292, 2004.

[30] J.A. Stratton and L.J. Chu, Diffraction theory of electromagnetic waves, Phys. Rev., 56(1), 99, 1939.

[31] Stephanos Venakides, Mansoor A. Haider and Vassilis Papanicolaou, Boundary integral calculations of two-dimensional electromagnetic scattering by photonic crystal Fabry-Perot structures, SIAM J. Appl. Math., 60(5), 1686-1706, (electronic) 2000.

[32] R.W. Wood, On the remarkable case of uneven distribution of a light in a diffractived grating spectrum, Philo. Mag., 4, 396-402, 1902.

[33] Pasi Ylä-Oijala and Matti Taskinen, Well-conditioned Müller formulation for electromagnetic scattering by dielectric objects, IEEE Trans. Antennas and Propagation, 53, no. 10, 33163323, 2005.

[34] V.V. Zalipaev and M.M. Popov, Wood's anomalies in a problem of the diffraction of a planar wave by a smooth periodic boundary, Zap. Nauchn. Sem. Leningrad. Otdel. Mat. Inst. Steklov. (LOMI), 186, 1990, no. Mat. Vopr. Teor. Rasprostr. Voln., 20, 87-100, 182. 